\title{
Synthesis, Antifungal Evaluation and Molecular Docking Studies of Some Tetrazole Derivatives
}

\section{Mohammad Hosein Afsarian, ${ }^{1}$ Mojtaba Farjam, ${ }^{2,3, \star}$ Elham Zarenezhad, ${ }^{2, \star}$ Somayeh Behrouz ${ }^{4}$ and Mohammad Navid Soltani Rad ${ }^{4}$}

\author{
${ }^{1}$ Department of Medical Mycology \& Parasitology, School of Medicine, Fasa University of Medical Sciences, Fasa, Iran \\ ${ }^{2}$ Noncommunicable Diseases Research Center, School of Medicine, Fasa University of Medical Sciences, Fasa, Iran \\ ${ }^{3}$ Department of Medical pharmacology, school of medicine, Fasa University of medical sciences, Fasa, Iran \\ ${ }^{4}$ Medicinal Chemistry Research Laboratory, Department of Chemistry, Shiraz University of Technology, Shiraz, Iran \\ *Corresponding author: E-mail: El.Zarenezhad@gmail.com \\ farjam.md@gmail.com; mfarjam@fums.ac.ir
}

Received: 01-25-2019

\begin{abstract}
A facile and simple protocol for the [3+2] cycloaddition of alkyl nitriles (RCN) with sodium azide $\left(\mathrm{NaN}_{3}\right)$ in the presence of copper bis(diacetylcurcumin) 1,2-diaminobenzene Schiff base complex, $\mathrm{SiO}_{2}-[\mathrm{Cu}-\mathrm{BDACDABSBC}]$ as a heterogeneous catalyst in the presence of ascorbic acid and a solution of water/i-PrOH (50:50, V/V) media at reflux condition is described. The supported catalyst was prepared by immobilization of a copper bis(diacetylcurcumin) 1,2-diaminobenzene Schiff base complex [Cu-BDACDABSBC] on silica gel. The complex has high selectivity, catalytic activity, and recyclability. The significant features of this procedure are high yields, broad substrate scope and simple and efficient work-up procedure. According to this synthetic methodology, excellent yields of 5 -substituted $1 \mathrm{H}$-tetrazoles having bioactive $\mathrm{N}$-heterocyclic cores were synthesized. The in vitro antifungal activities of title compounds were screened against various pathogenic fungal strains, such as Candida species

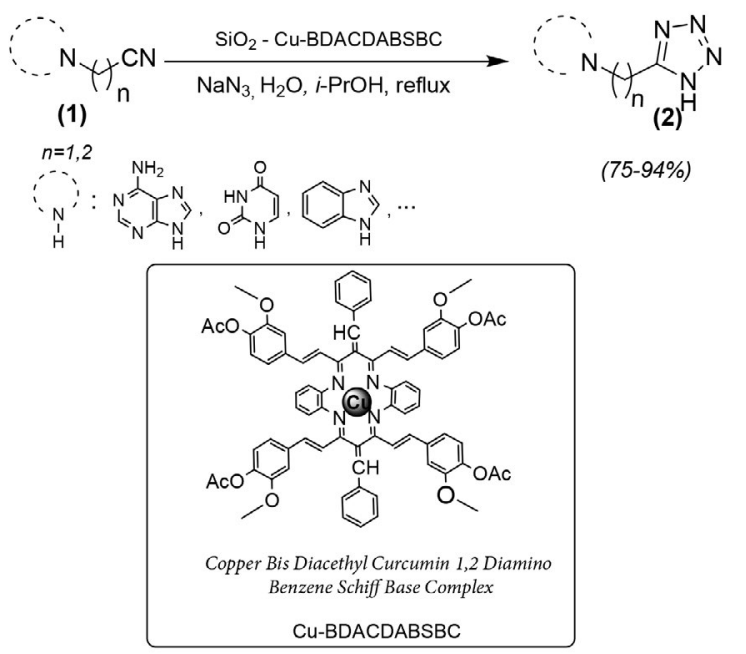
involving C. albicans, C. glabrata, C. krusei, C. parapsilosis as well as filamentous fungi like Aspergillus species consisting of A. fumigatus and A. flavus. The molecular docking analysis is discussed for one most potent compound against fungi. The docking study determined a remarkable interaction between the most potent compounds and the active site of $M y$ cobacterium P450DM.
\end{abstract}

Keywords: 'Click' cycloaddition; tetrazoles; heterogeneous catalyst; antifungal activity; docking studies.

\section{Introduction}

Tetrazole and its derivatives have attracted considerable interest in recent years because of their unique structure and wide range of applications. ${ }^{1}$ Heterocyclic com- pounds exhibiting tetrazole structures are known as bioactive compounds, encompassing a broad spectrum of biological activities such as antihypertensive, ${ }^{2}$ antibacterial, ${ }^{3,4}$ antifungal, ${ }^{5}$ anticonvulsant, ${ }^{6}$ analgesics, ${ }^{7}$ anti-inflammatory, ${ }^{8}$ antitubercular, ${ }^{9}$ anticancer, ${ }^{10}$ antineoplastic, ${ }^{11}$ antial- 


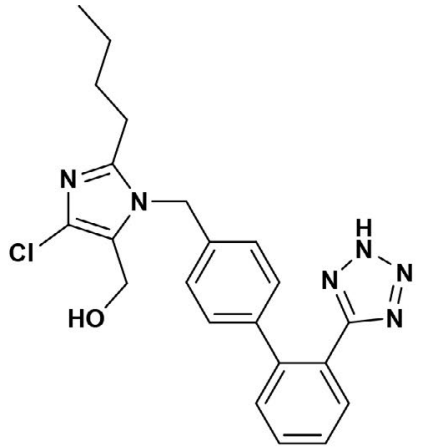

Losartan

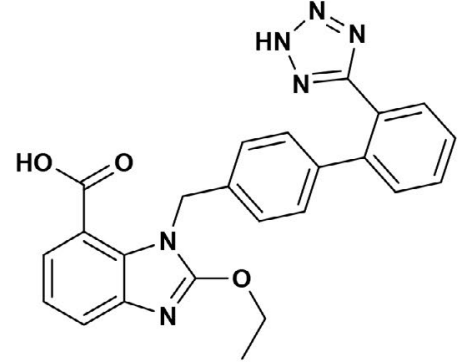

candesartan<smiles>Cc1nc(-[n+]2nc(-c3ccccc3)nn2-c2ccccc2)sc1C</smiles>

MTT

Figure 1. Structure of some of known tetrazol derivatives

lergic, ${ }^{12}$ antiviral, ${ }^{13}$ and especially anti-HIV activities. ${ }^{14}$ They play important roles in coordination chemistry as ligands, ${ }^{15}$ in medicinal chemistry as lipophilic spacers and metabolically stable surrogates for the carboxylic acid group and cis-amide bond, ${ }^{16}$ also in the photographic industry, ${ }^{17}$ in agriculture as plant growth regulators. ${ }^{18}$ This synthetic heterocyclic nitrogen-rich compounds are also useful synthons in synthetic organic chemistry. ${ }^{19}$ In addition, the syntheses of tetrazole moieties are very essential in modern medicinal chemistry, since they can behave as bioisosters for carboxylate moieties. Angiotensin (II) blockers often contain tetrazole cores, as losartan and candesartan. ${ }^{20}$ 3-(4,5-Dimethylthiazol-2-yl)-2,5-diphenyltetrazolium bromid (MTT) is a well-known tetrazole useful for evaluating cell metabolic activity (Figure 1). ${ }^{21,22}$

The different preparative methods for synthesis of tetrazoles are well documented. The traditional synthesis of 5-substituted $1 \mathrm{H}$-tetrazoles is conducted via [3+2] cycloaddition of azides to the corresponding nitriles, as developed by Sharpless and coworkers. ${ }^{23}$ However, several synthetic approaches have been determined so far that mostly proceed through the non-concerted types of mechanisms. ${ }^{24}$ Many homogeneous and heterogeneous catalysts were developed for the synthesis of tetrazoles such as $\mathrm{Cu}_{2} \mathrm{O},{ }^{25} \mathrm{AlCl}_{3}, \mathrm{BF}_{3} \cdot \mathrm{OEt}_{2},{ }^{27} \mathrm{Pd}\left(\mathrm{PPh}_{3}\right)_{4},{ }^{28} \mathrm{Yb}(\mathrm{OTf})_{3},{ }^{29}$ $\mathrm{Zn}(\mathrm{OTf})_{3},{ }^{30} \mathrm{CuSO}_{4},{ }^{31}$ and boron azides, ${ }^{32}$ also several heterogeneous catalyst systems, such as silica-supported $\mathrm{FeCl}_{3},{ }^{33} \mathrm{ZnS}$ nanospheres, ${ }^{34}$ zinc hydroxyapatite, ${ }^{35} \mathrm{Cu}-\mathrm{Zn}$ alloy nanopowder, ${ }^{36} \mathrm{Zn} / \mathrm{Al}$ hydrotalcite, ${ }^{37} \mathrm{Sb}_{2} \mathrm{O}_{3},{ }^{38}$ metal tungstates, ${ }^{39} \mathrm{CdCl}_{2},{ }^{40} \gamma$ - $\mathrm{Fe}_{2} \mathrm{O}_{3},{ }^{41}$ and natural natrolite zeolite ${ }^{42}$ were reported.

The copper-based complexes have undeniable roles in 'Click' chemistry; so far various solid compounds were used to support the copper species containing alumina, charcoal, silica gel and zeolites. Recently, the improvement of complexes supported on silica gel has received a great attention, ${ }^{43}$ because silica gel as an inorganic support has a high surface area $\left(5-800 \mathrm{~m}^{2} \mathrm{~kg}^{-1}\right)$ compared with other inorganic supports and silica gel consequently ranks at the top of the list of solids with high-surface areas. ${ }^{44-46}$ The development of complexes supported on silica gel has received considerable attention, because industry seeks more eco-friendly chemical manufacturing processes. ${ }^{47-49}$

Immobilization of organometallic complexes or homogenous catalysts on inorganic supports seems to be an appropriate approach to improve their stability, reactivity and selectivity. This method obtained the remarkable attention due to the ability of both, to facilitate the catalyst separation and its recycling. ${ }^{50-56}$

The chemical structure of curcumin was discovered by Milobedzka and coworkers. Curcumin as a tautomeric form is diarylheptanoic compound, which represents natural phenols responsible for turmeric's yellow color. ${ }^{57}$ The presence of the aromatic ring systems, which are phenols, and connected by two $\alpha, \beta$-unsaturated carbonyl groups allows the possibility of grafting with many biomolecules, organic and inorganic materials. Curcumin possesses very interesting pharmacological and biological properties exhibiting a variety of biological activities. ${ }^{58-59}$ The heterocyclic N,O-donor Schiff base ligands display a great role in the development of coordination chemistry because they easily form complexes with most of the transition metal ions. ${ }^{60-61}$

The incidence of opportunistic fungal infections has remarkably increased in recent years by normal flora fungi or acquired from the environment, especially Candida and Aspergillus species in immunocompromised or immunosuppressed patients. ${ }^{62-64}$ Increasing of opportunistic pathogenic fungal infections in these patients has become one of the most essential challenges for medicine. To date, it was observed that some of these fungi have become resistant to the established drugs, despite the introduction of new antifungal agents. ${ }^{65-66}$ Numerous antifungal drugs are available, such as: azoles (fluconazole, voriconazole and itraconazole) which are considered as the first-line therapy in current clinical use. ${ }^{67-69}$ However, the discovery of new antifungal drugs particularly for the treatment of opportunistic pathogenic fungal infections is critically essential.

Since the considerable therapeutic activities of $\mathrm{N}$-heterocyclic compounds ${ }^{70}$ were demonstrated and also 
in continuation of our interest in discovering copper(II) Schiff base complexes ${ }^{71-72}$ and the new $N$-heterocyclic bioactive compounds, ${ }^{73-79}$ herein we report the application of a suitable and reusable $\mathrm{Cu}(\mathrm{II})$-curcumin complex supported on silica gel and ascorbic acid as the reducing agent. This heterogeneous catalyst system exhibits a potent catalytic activity for the synthesis of some 5-substituated- $1 \mathrm{H}$-tetrazole derivatives tethered to bioactive $\mathrm{N}$-heterocyclic cores.

\section{Experimental}

\section{1. General}

All preliminary chemicals and solvents were purchased from Fluka or Merck. The catalyst was prepared according to the reported procedure. ${ }^{80}$ Reactions were monitored by TLC using SILG/UV 254 silica-gel plates. Column chromatography was performed on silica gel 60 (0.063-0.200 mm, 70-230 mesh; ASTM). IR spectra were measured using a Shimadzu FT-IR-8300 spectrophotometer. ${ }^{1} \mathrm{H}$ and ${ }^{13} \mathrm{C}$ NMR spectra were obtained on Bruker Avance-DPX-250/400 spectrometer operating at 250/62.5 and/or 400/100 MHz, respectively. Chemical shifts are given in $\delta$ relative to tetramethylsilane (TMS) as the internal standard, coupling constants $J$ are given in Hz. Abbreviations used for ${ }^{1} \mathrm{H}$ NMR signals are: $\mathrm{s}=$ singlet, $\mathrm{d}=$ doublet, $\mathrm{t}=$ triplet, $\mathrm{q}=$ quartet, $\mathrm{m}=$ multiplet, $\mathrm{br}=$ broad. Elemental analyses were performed on a Perkin-Elmer 240-B micro-analyzer.

\section{2. General Procedure for Immobilization of [Cu-BDACDABSBC] on Silica Gel}

To a solution of [Cu-BDACDABSBC] (1.28 g, 1 $\mathrm{mmol})$ in anhydrous dimethyl sulfoxide $(55 \mathrm{~mL})$, it was added a fresh and active silica gel $(0.6 \mathrm{~g}, 10 \mathrm{mmol})$ in $0.063-0.200 \mathrm{~mm}$ or $70-230$ mesh size, then the mixture was sonicated for $1 \mathrm{~h}$ and stirred at room temperature for $48 \mathrm{~h}$. Afterward, the resulting precipitate was filtered off and the solid residue (catalyst) was washed with dimethyl sulfoxide $(2 \times 50 \mathrm{~mL})$, methanol $(3 \times 50 \mathrm{~mL})$ and ether $(2$ $\times 50 \mathrm{~mL}$ ), dried in vacuum oven at $60^{\circ} \mathrm{C}$ for $4 \mathrm{~h}$ and stored in a refrigerator.

\section{3. General Procedure for the Preparation of Alkyl Nitriles 1a-o}

To a round bottom flask $(100 \mathrm{~mL})$, equipped with a condenser, was added $N$-heterocyclic compound (0.01 $\mathrm{mol})$, 3-chloroacetonitrile or 2-chloropropanenitrile $(0.013 \mathrm{~mol}), \mathrm{K}_{2} \mathrm{CO}_{3}(0.01 \mathrm{~mol}), \mathrm{Et}_{3} \mathrm{~N}(0.01 \mathrm{~mol})$, and a catalytic amount of TBAI $(0.1 \mathrm{~g})$ in anhydrous MeCN (40 $\mathrm{mL}$ ). The reaction mixture was refluxed until TLC monitoring indicated no further progress in the conversion. The solvent was evaporated in vacuo to remove the solvent. To continue, the remaining foam was dissolved in $\mathrm{CHCl}_{3}(100$ $\mathrm{mL})$ and subsequently washed with water $(2 \times 100 \mathrm{~mL})$. The organic layer was dried $\left(\mathrm{Na}_{2} \mathrm{SO}_{4}\right)$ and evaporated. The crude product was purified by column chromatography on silica gel. The catalyst was filtered off, washed with THF/ $\mathrm{H}_{2} \mathrm{O}(5 \times 10 \mathrm{~mL})$ and the filtrate was evaporated under vacuum to remove the solvent. The remaining foam was dissolved in $\mathrm{CHCl}_{3}(100 \mathrm{~mL})$ and subsequently washed with water $(2 \times 100 \mathrm{~mL})$. The organic layer was dried $(\mathrm{Na}-$ ${ }_{2} \mathrm{SO}_{4}$ ) and evaporated. The crude product was purified by column chromatography on silica gel and eluted with proper solvents.

\section{4. General Procedure for the Catalytic Test}

In a double-necked round bottom flask $(100 \mathrm{~mL})$ equipped with a condenser was added a mixture consisting of alkyl nitrile $(0.01 \mathrm{~mol}), \mathrm{NaN}_{3}(0.015 \mathrm{~mol})$, and $\mathrm{SiO}_{2^{-}}$ [Cu-BDACDABSBC] (0.05 mol \%) in $\mathrm{H}_{2} \mathrm{O} / i-\mathrm{PrOH}(1: 1$ $\mathrm{V} / \mathrm{V}, 50 \mathrm{~mL}$ ). The mixture was heated at reflux until TLC monitoring indicated no further improvement in the conversion (Table 4). The reaction mixture was then cooled to room temperature, vacuum-filtered and the residue was washed with ethyl acetate $(2 \times 20 \mathrm{~mL})$. To achieve $\mathrm{pH} 3$, the filtrate was treated with $5 \mathrm{~N} \mathrm{HCl}$ and stirred at room temperature for 30 minutes. Subsequently, the organic layer was separated, dried over anhydrous $\mathrm{Na}_{2} \mathrm{SO}_{4}$ and evaporated in vacuo. The crude product was purified by column chromatography on silica gel eluted with proper solvents and/or recrystallization was applied. Characterization data of all synthesized compounds are described below.

((1H-Tetrazol-5-yl)methyl)-2-methyl-1H-benzo[d] imidazole (2a)

Recrystallization (EtOAc) afforded a creamy solid; yield: $1.71 \mathrm{~g}(80 \%) ; \mathrm{mp}>300^{\circ} \mathrm{C}$ (dec.); $\mathrm{R}_{f}=0.25$ (EtOAc$\mathrm{MeOH}, 1: 1)$; IR (KBr): 3384, 3100, 2982, 1619, 1580, 1480 $\mathrm{cm}^{-1} ;{ }^{1} \mathrm{H}$ NMR $\left(250 \mathrm{MHz}, \mathrm{DMSO}-d_{6}\right): \delta 7.57-7.46(\mathrm{~m}, 2 \mathrm{H}$, aryl), 7.16-7.08 (m, 2H, aryl), $5.46\left(\mathrm{~s}, 2 \mathrm{H}, \mathrm{NCH}_{2}\right), 4.55$ (s, $1 \mathrm{H}$, exchangeable with $\mathrm{D}_{2} \mathrm{O}, \mathrm{NH}$, tetrazole), $2.67(\mathrm{~s}, 3 \mathrm{H}$, $\left.\mathrm{CH}_{3}\right) ;{ }^{13} \mathrm{C}$ NMR $\left(250 \mathrm{MHz}, \mathrm{DMSO}-d_{6}\right): \delta 17.90,49.18$, $115.53,116.64,121.56,123.05,134.72,140.35,151.27$, 158.41; MS (EI): $m / z$ (\%) $214(11.4)\left[\mathrm{M}^{+}\right]$. Anal. Calcd for $\mathrm{C}_{10} \mathrm{H}_{10} \mathrm{~N}_{6}$ : C, 56.07; H, 4.71; N, 39.23. Found: C, 56.19; H, $4.62 ; \mathrm{N}, 39.35$.

((1H-Tetrazol-5-yl)methyl)-1H-benzo[d]imidazole (2b) Recrystallization (EtOAc) afforded a yellow solid; yield: $1.80 \mathrm{~g}(90 \%) ; \mathrm{mp} 235-240{ }^{\circ} \mathrm{C}\left(\mathrm{dec}\right.$.); $\mathrm{R}_{f}=0.25$ (EtOAc-MeOH, 1:1); IR (KBr): 3385, 3100, 2968, 2800, 1616, $1462,1410 \mathrm{~cm}^{-1} ;{ }^{1} \mathrm{H}$ NMR $\left(250 \mathrm{MHz}\right.$, DMSO- $\left.d_{6}\right): \delta 8.28$ (s, $1 \mathrm{H}, \mathrm{C}(2)-\mathrm{H}$, benzimidazole), 7.64-7.61 ( $\mathrm{m}, 2 \mathrm{H}$, aryl), 7.22-7.13 (m, $2 \mathrm{H}$, aryl), $5.55\left(\mathrm{~s}, 2 \mathrm{H}, \mathrm{NCH}_{2}\right), 2.51(\mathrm{~s}, 1 \mathrm{H}$, exchangeable with $\mathrm{D}_{2} \mathrm{O}, \mathrm{NH}$, tetrazole); ${ }^{13} \mathrm{C}$ NMR (250 $\left.\mathrm{MHz}, \mathrm{DMSO}-d_{6}\right): \delta 51.70,116.65,117.85,122.22,123.25$, 
133.61, 137.50, 145.60, 155.69; MS (EI): $m / z$ (\%) 200 (14.5) $\left[\mathrm{M}^{+}\right]$. Anal. Calcd for $\mathrm{C}_{9} \mathrm{H}_{8} \mathrm{~N}_{6}$ : C, 53.99; H, 4.03; N, 41.98 . Found: C, 54.06; H, 4.15; N, 41.92.

((2-Methyl-4-nitro-1H-imidazol-1-yl)methyl)-1H-tetrazole (2c)

Column chromatography (silica gel, EtOAc- $\mathrm{MeOH}$, 1:1) afforded a brown solid; yield: $1.56 \mathrm{~g}(75 \%)$; $\mathrm{mp} 208-$ $212{ }^{\circ} \mathrm{C}$ (dec.); $\mathrm{R}_{f}=0.31$ (EtOAc-MeOH, 1:1); IR (KBr): $3350,3128,2900,1645,1500,1456,1300 \mathrm{~cm}^{-1} ;{ }^{1} \mathrm{H}$ NMR $\left(250 \mathrm{MHz}, \mathrm{DMSO}-d_{6}\right): \delta 8.30$ (s, $1 \mathrm{H}, \mathrm{C}(5)-\mathrm{H}$, imidazole), $5.40\left(\mathrm{~s}, 2 \mathrm{H}, \mathrm{NCH}_{2}\right), 4.40\left(\mathrm{~s}, 1 \mathrm{H}\right.$, exchangeable with $\mathrm{D}_{2} \mathrm{O}$, $\mathrm{NH}$, tetrazole), $2.43\left(\mathrm{~s}, 3 \mathrm{H}, \mathrm{CH}_{3}\right) ;{ }^{13} \mathrm{C}$ NMR $(250 \mathrm{MHz}$, DMSO- $\left.d_{6}\right): \delta 15.74,48.22,121.10,147.82,153.09,160.97$; MS (EI): $m / z(\%) 209(8.1)\left[\mathrm{M}^{+}\right]$. Anal. Calcd for $\mathrm{C}_{6} \mathrm{H}_{7} \mathrm{~N}_{7} \mathrm{O}_{2}: \mathrm{C}, 34.45 ; \mathrm{H}, 3.37 ; \mathrm{N}, 46.88$. Found: C, 34.38; $\mathrm{H}$, 3.42; N, 46.94 .

\section{((2-Phenyl-1H-imidazol-1-yl)methyl)-1H-tetrazole (2d)}

Recrystallization (EtOAc) afforded a bright brown solid; yield: $1.92 \mathrm{~g}(85 \%) ; \mathrm{mp} 216-220^{\circ} \mathrm{C}\left(\mathrm{dec}\right.$.); $\mathrm{R}_{f}=0.33$ (EtOAc-MeOH, 1:1); IR (KBr): 3280, 3150, 2937, 2850, $1653,1476 \mathrm{~cm}^{-1} ;{ }^{1} \mathrm{H}$ NMR $\left(250 \mathrm{MHz}\right.$, DMSO- $\left.d_{6}\right): \delta 7.94-$ $7.91(\mathrm{~m}, 2 \mathrm{H}$, aryl), 7.58-7.46 (m, 3H, aryl), $7.20(\mathrm{~s}, 1 \mathrm{H}$, $\mathrm{C}(4)-\mathrm{H}$, imidazole), 6.95 (s, 1H, C(5)- $\mathrm{H}$, imidazole), 5.28 $\left(\mathrm{s}, 2 \mathrm{H}, \mathrm{NCH}_{2}\right), 2.50\left(\mathrm{~s}, 1 \mathrm{H}\right.$, exchangeable with $\mathrm{D}_{2} \mathrm{O}, \mathrm{NH}$, tetrazole); ${ }^{13} \mathrm{C}$ NMR $\left(250 \mathrm{MHz}, \mathrm{DMSO}-d_{6}\right): \delta 49.07$, $121.03,125.54,127.07,127.46,129.68,131.07,152.18$, 160.14; MS (EI): $m / z(\%) 226(17.3)\left[\mathrm{M}^{+}\right]$. Anal. Calcd for $\mathrm{C}_{11} \mathrm{H}_{10} \mathrm{~N}_{6}$ : C, 58.40; H, 4.46; N, 37.15. Found: C, 58.31; H, $4.58 ; \mathrm{N}, 37.02$.

((1H-Tetrazol-5-yl)methyl)-1,3-dimethyl-1H-purine2,6(3H,7H)-dione (2e)

Recrystallization (EtOAc- $\mathrm{MeOH}$ ) afforded a brown solid; yield: $2.46 \mathrm{~g}$ (94\%); $\mathrm{mp}>300{ }^{\circ} \mathrm{C}$ (dec.); $\mathrm{R}_{f}=0.27$ (EtOAc-MeOH, 1:1); IR (KBr): 3391, 2996, 1720, 1705, 1690, 1650, $1375 \mathrm{~cm}^{-1} ;{ }^{1} \mathrm{H}$ NMR $\left(250 \mathrm{MHz}\right.$, DMSO- $\left.d_{6}\right): \delta$ $8.38\left(\mathrm{~s}, 1 \mathrm{H}\right.$, exchangeable with $\mathrm{D}_{2} \mathrm{O}, \mathrm{NH}$, tetrazole), 7.39 (s, $1 \mathrm{H}, \mathrm{C}(8)-\mathrm{H}$, theophylline), $5.04\left(\mathrm{~s}, 2 \mathrm{H}, \mathrm{NCH}_{2}\right), 2.59$ (s, $\left.3 \mathrm{H}, \mathrm{N}(1)-\mathrm{CH}_{3}\right), 2.31\left(\mathrm{~s}, 3 \mathrm{H}, \mathrm{N}(3)-\mathrm{CH}_{3}\right) ;{ }^{13} \mathrm{C}$ NMR (250 MHz, DMSO- $\left.d_{6}\right): \delta 27.30,31.18,47.34,104.91,144.41$, 149.32, 151.57, 154.19, 159.10; MS (EI): $m / z$ (\%) 263 (10.7) $\left[\mathrm{M}^{+}\right]$. Anal. Calcd for $\mathrm{C}_{9} \mathrm{H}_{10} \mathrm{~N}_{8} \mathrm{O}_{2}: \mathrm{C}, 41.22 ; \mathrm{H}, 3.84 ; \mathrm{N}$, 42.73. Found: C, $41.28 ; \mathrm{H}, 3.80 ; \mathrm{N}, 42.81$.

((1H-Tetrazol-5-yl)methyl)pyrimidine-2,4(1H,3H)-dione (2f)

Recrystallization (EtOAc) afforded a creamy solid; yield: $1.55 \mathrm{~g}(80 \%)$; $\mathrm{mp} 285-290^{\circ} \mathrm{C}$; $\mathrm{R}_{f}=0.09$ (EtOAc$\mathrm{MeOH}, 1: 1)$; IR (KBr): 3365, 3129, 2876, 1723, 1706, $1650,1458 \mathrm{~cm}^{-1} ;{ }^{1} \mathrm{H}$ NMR $\left(250 \mathrm{MHz}, \mathrm{DMSO}-d_{6}\right): \delta 11.37$ (s, $1 \mathrm{H}$, exchangeable with $\mathrm{D}_{2} \mathrm{O}, \mathrm{NH}$, uracil), $7.67(\mathrm{~d}, 1 \mathrm{H}$, $J=7.5 \mathrm{~Hz}, \mathrm{C}(6)-\mathrm{H}$, uracil), 5.70 (d, $1 \mathrm{H}, J=7.5 \mathrm{~Hz}, \mathrm{C}(5)-\mathrm{H}$, uracil), $5.07\left(\mathrm{~s}, 2 \mathrm{H}, \mathrm{NCH}_{2}\right), 4.07(\mathrm{~s}, 1 \mathrm{H}$, exchangeable with $\mathrm{D}_{2} \mathrm{O}, \mathrm{NH}$, tetrazole); ${ }^{13} \mathrm{C} \mathrm{NMR}(250 \mathrm{MHz}$,
DMSO- $\left.d_{6}\right): \delta 47.32,103.40,142.15,151.57,156.46$, 161.72; MS (EI): $m / z(\%) 194(10.8)\left[\mathrm{M}^{+}\right]$. Anal. Calcd for $\mathrm{C}_{6} \mathrm{H}_{6} \mathrm{~N}_{6} \mathrm{O}_{2}:$ C, 37.12; $\mathrm{H}, 3.11 ; \mathrm{N}, 43.29$. Found: C, 37.24; $\mathrm{H}, 3.26$; N, 43.24 .

\section{((1H-Tetrazol-5-yl)methyl)-9H-purin-6-amine (2g)}

Recrystallization (EtOAc) afforded a creamy solid; yield: $1.71 \mathrm{~g}(79 \%) ; \mathrm{mp}>300{ }^{\circ} \mathrm{C}$ (dec.); $\mathrm{R}_{f}=0.25$ (EtOAc$\mathrm{MeOH}, 1: 1)$; IR (KBr): 3328, 3100, 2853, 1676, 1520, 1471 $\mathrm{cm}^{-1} ;{ }^{1} \mathrm{H}$ NMR $\left(250 \mathrm{MHz}, \mathrm{DMSO}-d_{6}\right): \delta 8.12(\mathrm{~s}, 1 \mathrm{H}$, $\mathrm{C}(8)-\mathrm{H}$, adenine), 8.05 (s, $1 \mathrm{H}, \mathrm{C}(2)-\mathrm{H}$, adenine), 7.17 (s, $2 \mathrm{H}$, exchangeable with $\left.\mathrm{D}_{2} \mathrm{O}, \mathrm{NH}_{2}\right), 5.42\left(\mathrm{~s}, 2 \mathrm{H}, \mathrm{NCH}_{2}\right)$, $4.80\left(\mathrm{~s}, 1 \mathrm{H}\right.$, exchangeable with $\mathrm{D}_{2} \mathrm{O}, \mathrm{NH}$, tetrazole); ${ }^{13} \mathrm{C}$ NMR $\left(250 \mathrm{MHz}, \mathrm{DMSO}-d_{6}\right): \delta 54.31,118.43,139.90$, 147.78, 151.57, 155.69, 162.84; MS (EI): $m / z$ (\%) 217 (9.5) $\left[\mathrm{M}^{+}\right]$. Anal. Calcd for $\mathrm{C}_{7} \mathrm{H}_{7} \mathrm{~N}_{9}$ : C, 38.71; H, 3.25; N, 58.04. Found: C, 38.63; H, 3.18; N, 58.12.

\section{((1H-Tetrazol-5-yl)methyl)isoindoline-1,3-dione (2h)}

Recrystallization (EtOAc) afforded a creamy solid; yield: $2.08 \mathrm{~g}$ (91\%); $\mathrm{mp} 245-249^{\circ} \mathrm{C}$; $\mathrm{R}_{f}=0.47$ (EtOAc$\mathrm{MeOH}, 1: 1)$; IR (KBr): 3370, 3068, 2981, 1766, 1700, 1660, $1495 \mathrm{~cm}^{-1} ;{ }^{1} \mathrm{H}$ NMR $\left(250 \mathrm{MHz}, \mathrm{DMSO}-d_{6}\right): \delta 7.90-7.85$ $\left(\mathrm{m}, 4 \mathrm{H}\right.$, aryl), $4.87\left(\mathrm{~s}, 2 \mathrm{H}, \mathrm{NCH}_{2}\right), 4.04(\mathrm{~s}, 1 \mathrm{H}$, exchangeable with $\mathrm{D}_{2} \mathrm{O}, \mathrm{NH}$, tetrazole); ${ }^{13} \mathrm{C}$ NMR $(250 \mathrm{MHz}$, DMSO- $\left.d_{6}\right): \delta 42.20,127.27,131.49,133.48,157.10,167.81$; MS (EI): $m / z$ (\%) 229 (15.9) $\left[\mathrm{M}^{+}\right]$. Anal. Calcd for $\mathrm{C}_{10} \mathrm{H}_{7} \mathrm{~N}_{5} \mathrm{O}_{2}$ : C, 52.40; H, 3.08; N, 30.56. Found: C, 52.48; $\mathrm{H}, 3.01 ; \mathrm{N}, 30.69$.

((1H-Tetrazol-5-yl)methyl)benzo[d]isothiazol-3(2H)one 1,1-dioxide (2i)

Recrystallization (EtOAc) afforded a pale-yellow solid; yield: $2.12 \mathrm{~g}$ (80\%); $\mathrm{mp} 225-229^{\circ} \mathrm{C}$ (dec.); $\mathrm{R}_{f}=0.18$ (EtOAc-MeOH, 1:1); IR (KBr): 3324, 3050, 2976, 1715, 1600, 1460, 1321, $761 \mathrm{~cm}^{-1}$; ${ }^{1} \mathrm{H}$ NMR $(250 \mathrm{MHz}$, DMSO- $\left.\mathrm{d}_{6}\right): \delta 7.84-7.45\left(\mathrm{~m}, 4 \mathrm{H}\right.$, aryl), $4.44\left(\mathrm{~s}, 2 \mathrm{H}, \mathrm{NCH}_{2}\right)$, $2.51\left(\mathrm{~s}, 1 \mathrm{H}\right.$, exchangeable with $\mathrm{D}_{2} \mathrm{O}, \mathrm{NH}$, tetrazole); ${ }^{13} \mathrm{C}$ NMR $\left(250 \mathrm{MHz}, \mathrm{DMSO}-d_{6}\right): \delta 39.52,126.32,126.88$, $127.33,131.63,132.06,139.30,156.66,169.10$. MS (EI): $m / z(\%) 265(19.7)\left[\mathrm{M}^{+}\right]$. Anal. Calcd for $\mathrm{C}_{10} \mathrm{H}_{8} \mathrm{~N}_{4} \mathrm{O}_{3} \mathrm{~S}: \mathrm{C}$, 45.45; H, 3.05; N, 21.20; S, 12.13. Found: C, 45.56; H, 3.11; $\mathrm{N}, 21.14 ; \mathrm{S}, 12.25$.

\section{(2-(2-Methyl-4-nitro-1H-imidazol-1-yl)ethyl)-1H-tet- razole $(2 \mathbf{j})$}

Column chromatography (silica gel, EtOAc- $\mathrm{MeOH}$, 1:1) afforded a creamy solid; yield: $1.74 \mathrm{~g}(78 \%)$; mp 210$215^{\circ} \mathrm{C} ; \mathrm{R}_{f}=0.23$ (EtOAc-MeOH, 1:1); IR (KBr): 3358, 3100, 2965, 1653, 1525, 1460, $1345 \mathrm{~cm}^{-1} ;{ }^{1} \mathrm{H}$ NMR (250 $\left.\mathrm{MHz}, \mathrm{DMSO}-d_{6}\right): \delta 8.26(\mathrm{~s}, 1 \mathrm{H}, \mathrm{C}(5)-\mathrm{H}$, imidazole), 4.27 $\left(\mathrm{t}, 2 \mathrm{H}, J=7.2 \mathrm{~Hz}, \mathrm{NCH}_{2}\right), 3.61(\mathrm{~s}, 1 \mathrm{H}$, exchangeable with $\mathrm{D}_{2} \mathrm{O}, \mathrm{NH}$, tetrazole), $3.12\left(\mathrm{t}, 2 \mathrm{H}, J=7.2 \mathrm{~Hz}, \mathrm{NCH}_{2} \mathrm{CH}_{2}\right)$, $2.21\left(\mathrm{~s}, 3 \mathrm{H}, \mathrm{CH}_{3}\right) ;{ }^{13} \mathrm{C}$ NMR $\left(250 \mathrm{MHz}, \mathrm{DMSO}-d_{6}\right): \delta$ $15.99,27.43,52.03,121.49,148.55,153.83,163.97$; MS (EI): $m / z(\%) 223(10.6)\left[\mathrm{M}^{+}\right]$. Anal. Calcd for 
$\mathrm{C}_{9} \mathrm{H}_{11} \mathrm{~N}_{5} \mathrm{O}_{2}$ : C, 48.86; H, 5.01; N, 31.66. Found: C, 48.94; $\mathrm{H}, 5.13 ; \mathrm{N}, 31.59$.

\section{5-(2-(2-Phenyl-1H-imidazol-1-yl)ethyl)-1H-tetrazole (2k)}

Recrystallization (EtOAc) afforded a creamy solid; yield: $1.92 \mathrm{~g}$ (80\%); $\mathrm{mp} 250-255^{\circ} \mathrm{C}$ (dec.); $\mathrm{R}_{f}=0.38$ (EtOAc-MeOH, 1:1); IR (KBr): 3300, 3050, 2960, 1650, $1485 \mathrm{~cm}^{-1}$; ${ }^{1} \mathrm{H}$ NMR $\left(250 \mathrm{MHz}\right.$, DMSO- $\left.d_{6}\right): \delta 7.58-7.44$ (m, 5H, aryl), 7.32 (s, 1H, C(4)-H), 6.95 (s, 1H, C(5)-H), $4.29\left(\mathrm{t}, 2 \mathrm{H}, J=7.5 \mathrm{~Hz}, \mathrm{NCH}_{2}\right), 3.07(\mathrm{t}, 2 \mathrm{H}, J=7.5 \mathrm{~Hz}$, $\left.\mathrm{NCH}_{2} \mathrm{CH}_{2}\right), 1.98\left(\mathrm{~s}, 1 \mathrm{H}\right.$, exchangeable with $\mathrm{D}_{2} \mathrm{O}, \mathrm{NH}$, tetrazole); ${ }^{13} \mathrm{C}$ NMR $\left(250 \mathrm{MHz}, \mathrm{DMSO}-d_{6}\right): \delta 30.01,54.59$, $120.83,125.87,126.30,127.05,127.27,130.29,149.77$, 155.14; MS (EI): $m / z(\%) 240(15.8)\left[\mathrm{M}^{+}\right]$. Anal. Calcd for $\mathrm{C}_{12} \mathrm{H}_{12} \mathrm{~N}_{6}$ : C, 59.99; H, 5.03; N, 34.98. Found: C, 60.07; H, $5.16 ; \mathrm{N}, 34.87$.

\section{(2-(1H-Tetrazol-5-yl)ethyl)-1 $H$-benzo $[d]$ imidazole (2l)}

Recrystallization (EtOAc) afforded a brown solid; yield: $1.79 \mathrm{~g}$ (84\%); $\mathrm{mp} 300-304{ }^{\circ} \mathrm{C}$ (dec.); $\mathrm{R}_{f}=0.70$ (EtOAc-MeOH, 1:1); IR (KBr): 3326, 3100, 2926, 1653, $1501,1470 \mathrm{~cm}^{-1} ;{ }^{1} \mathrm{H}$ NMR $\left(250 \mathrm{MHz}, \mathrm{DMSO}-d_{6}\right): \delta 8.10$ (s, $1 \mathrm{H}, \mathrm{C}(2)-\mathrm{H}$, benzimidazole), 7.62-7.55 (m, $2 \mathrm{H}$, aryl), 7.24-7.14 (m, 2H, aryl), $4.55\left(\mathrm{t}, 2 \mathrm{H}, J=6.5 \mathrm{~Hz}, \mathrm{NCH}_{2}\right)$, $3.17\left(\mathrm{t}, 2 \mathrm{H}, J=6.5 \mathrm{~Hz}, \mathrm{NCH}_{2} \mathrm{CH}_{2}\right), 2.51(\mathrm{~s}, 1 \mathrm{H}$, exchangeable with $\mathrm{D}_{2} \mathrm{O}, \mathrm{NH}$, tetrazole); ${ }^{13} \mathrm{C}$ NMR $(250 \mathrm{MHz}$, DMSO- $\left.d_{6}\right): \delta 31.15,60.59,116.19,117.33,124.12,125.22$, 134.64, 138.76, 149.31, 161.72; MS (EI): $m / z(\%) 214$ (12.7) $\left[\mathrm{M}^{+}\right]$. Anal. Calcd for $\mathrm{C}_{10} \mathrm{H}_{10} \mathrm{~N}_{6}: \mathrm{C}, 55.94 ; \mathrm{H}, 4.63 ; \mathrm{N}$, 39.14. Found: C, 55.83; H, 4.75; N, 39.28.

\section{2-(2-(1H-Tetrazol-5-yl)ethyl)isoindoline-1,3-dione (2m)}

Recrystallization (EtOAc) afforded a creamy solid; yield: $2.11 \mathrm{~g}(87 \%)$; $\mathrm{mp}>300{ }^{\circ} \mathrm{C}\left(\mathrm{dec}\right.$.); $\mathrm{R}_{f}=0.70$ (EtOAc$\mathrm{MeOH}, 1: 1)$; IR (KBr): 3374, 3063, 2950, 1772, 1620, $1510,1458 \mathrm{~cm}^{-1} ;{ }^{1} \mathrm{H}$ NMR $\left(250 \mathrm{MHz}\right.$, DMSO- $\left.d_{6}\right): \delta 7.28-$ $7.09\left(\mathrm{~m}, 4 \mathrm{H}\right.$, aryl), $4.34\left(\mathrm{t}, 2 \mathrm{H}, J=7.2 \mathrm{~Hz}, \mathrm{NCH}_{2}\right), 3.46(\mathrm{~s}$, $1 \mathrm{H}$, exchangeable with $\mathrm{D}_{2} \mathrm{O}, \mathrm{NH}$, tetrazole), $3.08(\mathrm{t}, 2 \mathrm{H}, J$ $\left.=7.2 \mathrm{~Hz}, \mathrm{NCH}_{2} \mathrm{CH}_{2}\right) ;{ }^{13} \mathrm{C}$ NMR $\left(250 \mathrm{MHz}\right.$, DMSO- $\left.d_{6}\right): \delta$ 27.64, 42.69, 126.35, 131.64, 134.28, 159.48, 167.02; MS (EI): $m / z(\%) 243(13.9)\left[\mathrm{M}^{+}\right]$. Anal. Calcd for $\mathrm{C}_{11} \mathrm{H}-$ ${ }_{9} \mathrm{~N}_{5} \mathrm{O}_{2}: \mathrm{C}, 54.32 ; \mathrm{H}, 3.73 ; \mathrm{N}, 28.79$. Found: C, 54.43; $\mathrm{H}$, $3.82 ; \mathrm{N}, 28.86$.

\section{1-(2-(1H-Tetrazol-5-yl)ethyl)-4-phenylpiperazine (2n)}

Column chromatography (silica gel, EtOAc- $n$-hexane, 1:1) afforded a bright brown solid; yield: $2.35 \mathrm{~g}$ (91\%); $\mathrm{mp}>300{ }^{\circ} \mathrm{C}$ (dec.); $\mathrm{R}_{f}=0.23$ (EtOAc-MeOH, 1:1); IR (KBr): 3340, 3100, 2992, 1659, 1653, $1476 \mathrm{~cm}^{-1} ;{ }^{1} \mathrm{H}$ NMR $\left(250 \mathrm{MHz}, \mathrm{DMSO}-d_{6}\right): \delta 7.26-7.20(\mathrm{~m}, 2 \mathrm{H}$, aryl), 6.91$6.81(\mathrm{~m}, 3 \mathrm{H}$, aryl $), 4.96\left(\mathrm{~s}, 1 \mathrm{H}\right.$, exchangeable with $\mathrm{D}_{2} \mathrm{O}$, $\mathrm{NH}$, tetrazole), $3.83\left(\mathrm{t}, 2 \mathrm{H}, J=6.5 \mathrm{~Hz}, \mathrm{CH}_{2}\right), 3.09(\mathrm{t}, 2 \mathrm{H}, J$ $\left.=7.2 \mathrm{~Hz}, \mathrm{CH}_{2}\right), 2.72-2.65\left(\mathrm{~m}, 8 \mathrm{H}, 4 \mathrm{CH}_{2}\right) ;{ }^{13} \mathrm{C} \mathrm{NMR}(250$ MHz, DMSO- $\left.d_{6}\right): \delta 28.22,49.15,52.27,56.11,114.32$, $119.58,130.51,150.05,160.23$; MS (EI): $m / z$ (\%) 258 (19.7)
$\left[\mathrm{M}^{+}\right]$. Anal. Calcd for $\mathrm{C}_{13} \mathrm{H}_{18} \mathrm{~N}_{6}: \mathrm{C}, 60.44 ; \mathrm{H}, 7.02 ; \mathrm{N}$, 32.53. Found: C, $60.31 ; \mathrm{H}, 7.08 ; \mathrm{N}, 32.61$.

7-(2-(1H-Tetrazol-5-yl)ethyl)-1,3-dimethyl-1H-purine2,6(3H,7H)-dione (2o)

Column chromate-graphy (silica gel, $\mathrm{MeOH}$ ) afforded a brown foam; yield: $2.34 \mathrm{~g}(85 \%) ; \mathrm{R}_{f}=0.15$ (EtOAc$\mathrm{MeOH}, 1: 1)$; IR (KBr): 3300, 2985, 1716, 1702, 1693, 1658, $1379 \mathrm{~cm}^{-1} ;{ }^{1} \mathrm{H}$ NMR $\left(250 \mathrm{MHz}, \mathrm{DMSO}-d_{6}\right): \delta 7.86(\mathrm{~s}, 1 \mathrm{H}$, $\mathrm{C}(8)-\mathrm{H}$, theophylline), $4.55\left(\mathrm{t}, 2 \mathrm{H}, J=5.7 \mathrm{~Hz}, \mathrm{NCH}_{2}\right), 3.41$ $\left(\mathrm{t}, 2 \mathrm{H}, J=5.7 \mathrm{~Hz}, \mathrm{NCH}_{2} \mathrm{CH}_{2}\right), 3.27\left(\mathrm{~s}, 3 \mathrm{H}, \mathrm{N}(1)-\mathrm{CH}_{3}\right), 3.09$ $\left(\mathrm{s}, 3 \mathrm{H}, \mathrm{N}(3)-\mathrm{CH}_{3}\right), 2.54\left(\mathrm{~s}, 1 \mathrm{H}\right.$, exchangeable with $\mathrm{D}_{2} \mathrm{O}$, $\mathrm{NH}$, tetrazole); ${ }^{13} \mathrm{C}$ NMR $\left(250 \mathrm{MHz}\right.$, DMSO- $\left.d_{6}\right): \delta 20.17$, $31.08,32.38,34.54,106.39,136.89,145.91,147.79,154.58$, 159.86; MS (EI): $m / z$ (\%) 276 (20.7) [M+]. Anal. Calcd for $\mathrm{C}_{10} \mathrm{H}_{12} \mathrm{~N}_{8} \mathrm{O}_{2}$ : C, 43.48; $\mathrm{H}, 4.38 ; \mathrm{N}, 40.56$. Found: $\mathrm{C}, 43.59$; $\mathrm{H}, 4.27 ; \mathrm{N}, 40.61$.

\section{Results and Discussion}

\section{1. Chemistry of Synthesized Compound}

Initially, we prepared an active catalyst according to the procedure reported in the literature ${ }^{80}$ as shown in Scheme 1. First, curcumin (3) and acetic anhydride (4) were stirred in dry pyridine, the yellow crude product was collected and recrystallized from EtOAc/n-hexane $(60 / 40)$ to give diacetylcurcumin (DAC (5)) in 95\% yield. Then, DAC (452 mg, $1 \mathrm{mmol}$ ) and benzaldehyde (108 $\mathrm{mg}, 1 \mathrm{mmol}$ ) were dissolved in alcoholic media in the presence of a catalytic amount of piperidine and which readily afforded Ben-acetyl-curcumin ligand (6) in a good yield (76\%). Afterwards, for preparation of $\mathrm{Cu}^{(\mathrm{II})}$ complex 7 , in a conical flask, copper(II) chloride (1 $\mathrm{mmol})$ and orthophenylenediamine $(109 \mathrm{~mL}, 1 \mathrm{mmol})$ was refluxed in methanol for about $3 \mathrm{~h}$. To the above solution, benzilidene-acetyl curcumin $(574 \mathrm{mg}, 1 \mathrm{mmol})$ (6) in methanol was added and the contents were stirred for 24 hour. The microcrystalline product formed, after filtering, the crude was washed with methanol and dried in vacuo. After synthesis and supporting the catalyst on silica gel, we applied this heterogeneous catalyst in synthesis of some 1,2,3-triazolyl carbocyclic nucleoside derivatives.

In this methodology, the diverse $\mathrm{N}$-heterocycles including imides, xanthines, azoles, purine and pyrimidine nucleobases react with 3-chloropropanenitrile or 2-chloroacetonitrile, in the presence of an equimolar mixture of triethylamine and potassium carbonate (TEA) $-\mathrm{K}_{2} \mathrm{CO}_{3}$ as the base, and catalytic amount of tetrabutylammonium iodide (TBAI) in acetonitrile at reflux conditions to afford nitriles 1 . In continuation, these nitriles were able to perform the [3+2] cycloaddition reaction with sodium azide using $\mathrm{SiO}_{2}$-[Cu-BDACDABSBC] as the catalyst, and ascorbic acid as the reducing agent, in water-isopropanol media at reflux condition to yield tetrazoles $\mathbf{2}$. In most cas- 
<smiles>COc1cc(/C=C/C(=O)CC(=O)/C=C/c2ccc(O)c(OC)c2)ccc1O</smiles>

3<smiles>CC(=O)OC(C)=O</smiles>

4<smiles>COc1cc(/C=C/C(=O)/C=C/C(=O)/C=C/c2ccc(OC(C)=O)c(OC)c2)ccc1OC(C)=O</smiles>

(Ben-acetyl-curcumin ligand) $78 \%$

6

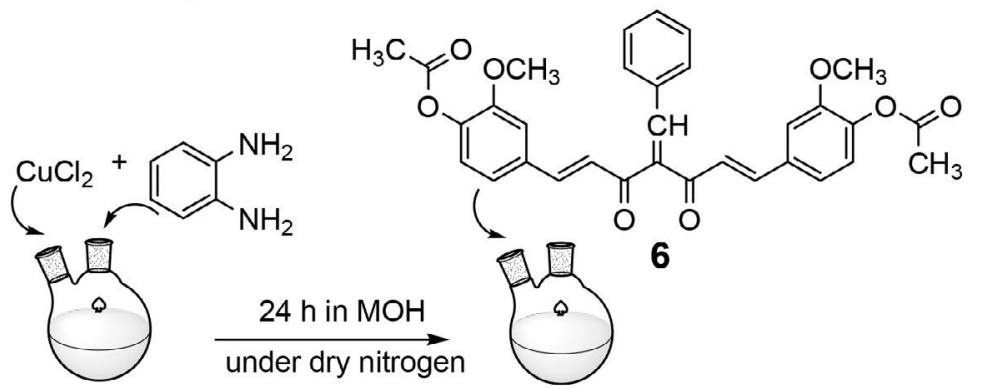

\section{Reflux $3 \mathrm{~h}$ in $\mathrm{MOH}$}

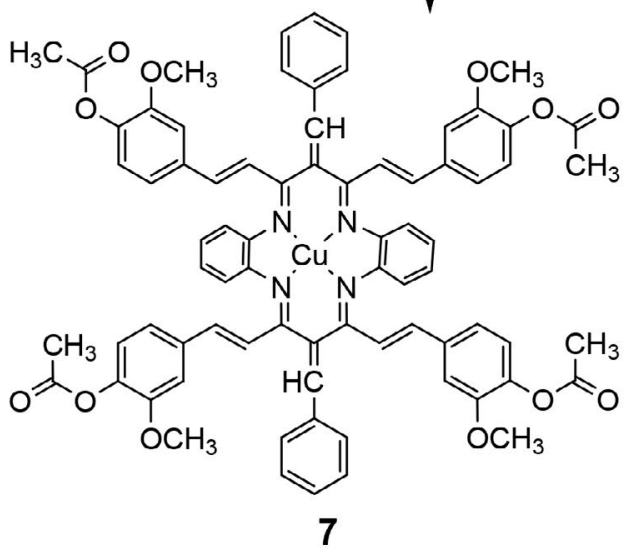<smiles>COc1cc(/C=C/C(=O)CC(=O)/C=C/c2ccc(OC(C)=O)c(OC)c2)ccc1OC(C)=O</smiles>

DAC: Diacetylcurcumin Recrystallize ETOAC/hexane (60/40) 95\%

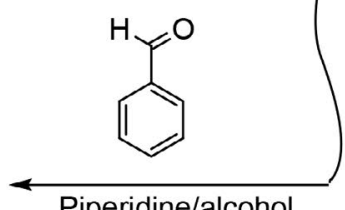

Piperidine/alcohol

Scheme 1. Preparation of $\mathrm{SiO}_{2}$ - $[\mathrm{Cu}-\mathrm{BDACDABSBC}]$.

es, the $N$-alkylation reactions were completed after refluxing for $48 \mathrm{~h}$ (Scheme 2).

The first step of this synthetic approach was represented by optimization of the reaction conditions. At first, we carried out the cycloaddition reaction of 2-(2-methyl-1H-benzo[d]imidazol-1-yl) acetonitrile (1a) and sodium azide as the model reaction to afford the $1-((1 H$-tetrazol-5-yl)methyl)-2-methyl-1H-benzo[ $d]$ imidazole (2a) (Table 1). The 1,3-dipolar cycloaddition of the model reaction was carried out in the presence of ascorbic acid (1 $\mathrm{mmol})$ and $\mathrm{SiO}_{2}-\mathrm{Cu}-\mathrm{BDACDABSBC}(0.05 \mathrm{~mol} \%)$ in $\mathrm{H}_{2} \mathrm{O}$ at different temperatures, which afforded $\mathbf{2 a}$ in $51 \%$ yield (as the best result) after refluxing for $5 \mathrm{~h}$ (Table 1 , entry 6 ).

To study the influence of temperature and $\mathrm{H}_{2} \mathrm{O}$, the model reaction was carried out at different temperatures (Table 1, entries 2-6). Due to Table 1, an increase in the temperature resulted in the promotion of cycloaddition reaction. The best result was obtained when the cycloaddition reaction was conducted at $100{ }^{\circ} \mathrm{C}$ for $5 \mathrm{~h}$ (Table 1 , entry 6). 


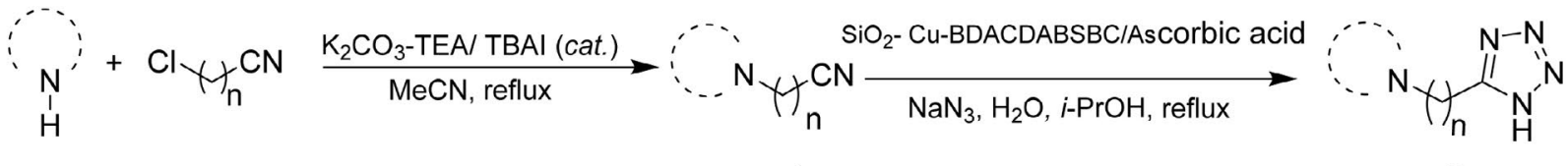

$n=1,2$

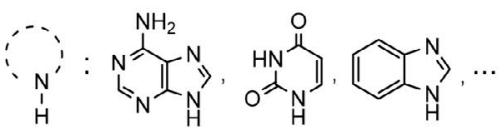

$(75-94 \%)$

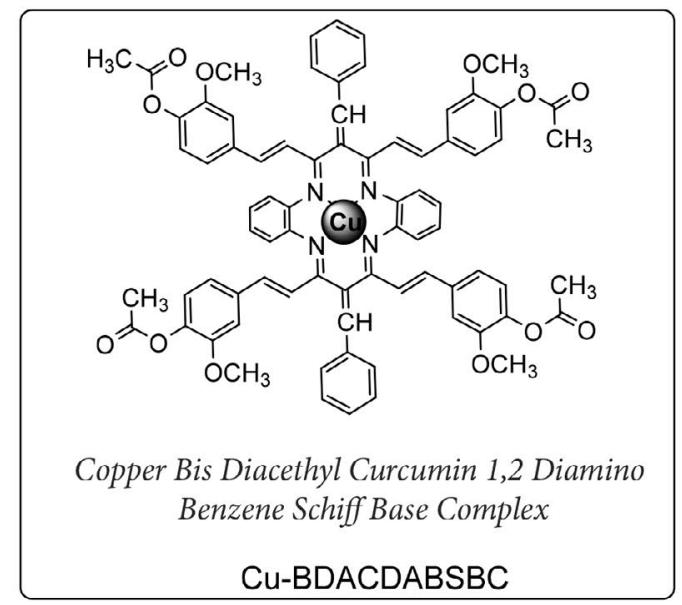

Scheme 2. Synthesis of 5 -substituated- $1 H$-tetrazole derivatives tethered to bioactive $N$-heterocyclic cores using $\mathrm{SiO}_{2}$-[Cu-BDACDABSBC] and ascorbic acid.

Table 1. Effect of $\mathrm{H}_{2} \mathrm{O}$ and temperature on [3+2] cycloaddition reaction of azide-nitrile using Cu-BDACDABSBC and ascorbic acid to afford $2 \mathbf{a}^{\mathrm{a}}$.

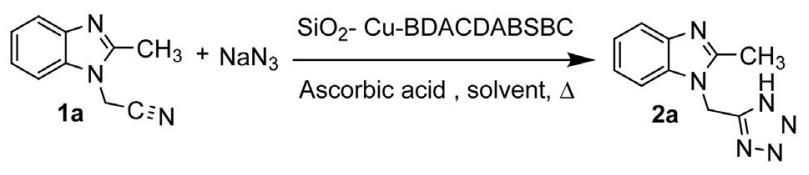

\begin{tabular}{ccccc}
\hline Entry & Solvent & $\mathbf{T}^{\circ} \mathbf{C}$ & Time (h) & Yield $^{\mathbf{b}}(\%)$ \\
\hline 1 & $\mathrm{H}_{2} \mathrm{O}$ & R.T. & 24 & 10 \\
2 & $\mathrm{H}_{2} \mathrm{O}$ & 50 & 10 & 20 \\
3 & $\mathrm{H}_{2} \mathrm{O}$ & 60 & 8 & 39 \\
4 & $\mathrm{H}_{2} \mathrm{O}$ & 70 & 8 & 38 \\
5 & $\mathrm{H}_{2} \mathrm{O}$ & 80 & 6 & 49 \\
6 & $\mathrm{H}_{2} \mathrm{O}$ & Reflux & 5 & 51 \\
\hline
\end{tabular}

${ }^{a}$ Reaction conditions: nitrile $(0.01 \mathrm{~mol}), \mathrm{NaN}_{3}(0.015 \mathrm{~mol})$, catalyst (0.05 mol \%), ascorbic acid (1 mmol), $\mathrm{H}_{2} \mathrm{O}(50 \mathrm{~mL}) .{ }^{\mathrm{b}}$ Isolated yield.

To further optimize the reaction conditions, the influence of various organic solvents/ $\mathrm{H}_{2} \mathrm{O}(\mathrm{V} / \mathrm{V})$ was examined in the presence of $\mathrm{SiO}_{2}$-Cu-BDACDABSBC $(0.05 \mathrm{~mol}$ $\%$ ) and ascorbic acid at various temperature (Table 2).

From Table 2 it is well demonstrated that the solvent has a significant role in the progress of the reaction. Among the examined solvents, a mixture of $i-\mathrm{PrOH}$ and water (Table 2, entry 1 ) afforded the best result, compared to pure water (Table 1, entry 6), The best ratio of $i-\mathrm{PrOH}$ to water for the progress of the reaction was observed to be $1: 1$ (Table 2, entry 2); however other ratios also yielded the product, albeit in lower amounts (Table 2, entries $3,4)$. Employing the other mixtures of solvents afforded a moderate yield of the product over longer periods of time
Table 2. Effect of solvent type and temperature on [3+2] cycloaddition reaction of azide-nitrile using $\mathrm{Cu}-\mathrm{BDACDABSBC}$ and ascorbic acid to afford $2 \mathbf{a}^{\mathrm{a}}$.

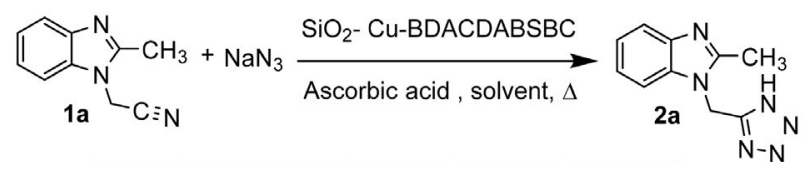

\begin{tabular}{|c|c|c|c|c|}
\hline Entry & Solvent & $\mathrm{T}^{\circ} \mathrm{C}$ & Time (h) & Yield ${ }^{\mathrm{b}}(\%)$ \\
\hline 1 & $\mathrm{H}_{2} \mathrm{O} / \mathrm{i}-\mathrm{PrOH}^{\mathrm{c}}$ & R.T. & 24 & 60 \\
\hline 2 & $\mathrm{H}_{2} \mathrm{O} / \mathrm{i}-\mathrm{PrOH}^{\mathrm{c}}$ & Reflux & 4 & 94 \\
\hline 3 & $\mathrm{H}_{2} \mathrm{O} / \mathrm{i}-\mathrm{PrOH}^{\mathrm{d}}$ & Reflux & 6 & 83 \\
\hline 4 & $\mathrm{H}_{2} \mathrm{O} / \mathrm{i}-\mathrm{PrOH}^{\mathrm{e}}$ & Reflux & 4 & 65 \\
\hline 5 & $\mathrm{H}_{2} \mathrm{O} / \mathrm{Me}_{2} \mathrm{CO}^{\mathrm{c}}$ & Reflux & 4 & 36 \\
\hline 6 & $\mathrm{H}_{2} \mathrm{O} / \mathrm{DMF}^{\mathrm{c}}$ & Reflux & 7 & 40 \\
\hline 7 & $\mathrm{H}_{2} \mathrm{O} / \mathrm{DMSO}^{c}$ & Reflux & 8 & 42 \\
\hline 8 & $\mathrm{H}_{2} \mathrm{O} / \mathrm{THF}^{\mathrm{c}}$ & Reflux & 8 & 60 \\
\hline 9 & $\mathrm{H}_{2} \mathrm{O} / \mathrm{HMPA}^{\mathrm{c}}$ & Reflux & 6 & 40 \\
\hline 10 & $\mathrm{H}_{2} \mathrm{O} / \mathrm{NMP}^{\mathrm{c}}$ & Reflux & 5 & 52 \\
\hline 11 & $\mathrm{H}_{2} \mathrm{O} /$ Toluene $^{\mathrm{c}, \mathrm{f}}$ & Reflux & 24 & 45 \\
\hline 12 & DMSO & 120 & 24 & 56 \\
\hline 13 & DMF & 120 & 24 & 58 \\
\hline 14 & THF & Reflux & 11 & 50 \\
\hline 15 & $\mathrm{i}-\mathrm{PrOH}$ & Reflux & 7 & 52 \\
\hline 16 & $\mathrm{EtOH}$ & Reflux & 5 & 45 \\
\hline
\end{tabular}

${ }^{a}$ Reaction conditions: nitrile $(0.01 \mathrm{~mol}), \mathrm{NaN}_{3}(0.015 \mathrm{~mol}), \mathrm{SiO}_{2}-$ $\mathrm{Cu}$-BDACDABSBC $(0.05 \mathrm{~mol} \%)$, solvent $(50 \mathrm{~mL}) .{ }^{\mathrm{b}}$ Isolated yield. ${ }^{c} 50: 50(\mathrm{~V} / \mathrm{V}) .{ }^{d} 70: 30(\mathrm{~V} / \mathrm{V}) .{ }^{\mathrm{e}}{ }^{30: 70}(\mathrm{~V} / \mathrm{V}) .{ }^{\mathrm{f}}$ In the presence of a catalytic amount $(0.16 \mathrm{~g}, 0.0005 \mathrm{~mol})$ of tetrabutylammonium bromide (TBAB)

(Table 2, entries 5-11). Additionally, when pure aprotic solvents such as DMSO, DMF, THF (Table 2, entries 1214) and $i-\mathrm{PrOH}$ and $\mathrm{EtOH}$ as protic solvents were used 
alone, moderate yields were obtained (Table 2, entries 15-16).

To investigate the catalytic potency of heterogeneous $\mathrm{SiO}_{2}-\mathrm{Cu}-\mathrm{BDACDABSBC}$ catalyst and other reported copper catalysts in cycloaddition reactions of azide-nitrile, the comparative results are summarized in Table 3. As shown in Table 3, when the reaction was carried out in the absence of a catalyst, this resulted in only marginal yield as indicated by GC analysis $(<\% 7)$, even if the reaction time was prolonged (Table 3 , entry 1 ).

Table 3. Comparing the catalytic potency of $\mathrm{SiO}_{2}-\mathrm{Cu}-$ BDACDABSBC with various catalysts. ${ }^{a}$

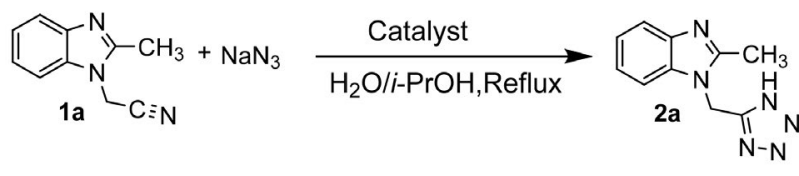

\begin{tabular}{ccccc}
\hline Entry & Catalyst & Time (h) & Yield $^{\mathbf{b}} \mathbf{( \% )}$ & Ref. \\
\hline 1 & - & 72 & $<8$ & - \\
2 & $\mathrm{Cu}_{2} \mathrm{O}$ & 10 & 64 & {$[25]$} \\
3 & $\mathrm{AlCl}_{3}$ & 10 & 76 & {$[26]$} \\
4 & $\mathrm{FeCl}_{3}-\mathrm{SiO}_{2}$ & 24 & 75 & {$[33]$} \\
5 & This catalyst & 4 & 94 & - \\
\hline
\end{tabular}

${ }^{a}$ Reaction conditions: nitrile $(0.01 \mathrm{~mol}), \mathrm{NaN}_{3}(0.015 \mathrm{~mol})$, catalyst, $\mathrm{H}_{2} \mathrm{O} / \mathrm{i}$-PrOH $(50 \mathrm{~mL}) .{ }^{b}$ Isolated yield. ${ }^{c} 0.05 \mathrm{~mol} \%$.

The potency of different reported heterogeneous or homogeneous catalysts in tetrazole synthesis was assessed (Table 3, entries 2-4). As shown in Table 3, higher yield of 2a were obtained and shorter reaction times were necessary when using heterogeneous $\mathrm{SiO}_{2}-\mathrm{Cu}-\mathrm{BDACDABSBC}$ catalyst (Table 3 , entry 5).

To illustrate the scope of this method, we extended the optimized reaction condition to the cycloaddition reaction of nitrile $1 \mathrm{a}$ with sodium azide (Table 4). As the results in Table 4 indicate, heterogeneous $\mathrm{SiO}_{2}$-Cu-BDACDABSBC catalyst proved to be useful catalyst for Huisgen cycloaddition between the structurally diverse $\beta$-azido alcohols and alkynes. All synthesized compounds $\mathbf{2 a}-\mathbf{o}$ were fully characterized, and their structures were confirmed by ${ }^{1} \mathrm{H}$ and ${ }^{13} \mathrm{C}$ NMR spectroscopy, elemental analysis, mass spectrometry and IR spectroscopy methods.

The reusability and recoverability of the $\mathrm{SiO}_{2}-\mathrm{Cu}$ BDACDABSBC catalyst on the sample reaction was studied during the synthesis of $\mathbf{2 a}$ (Table 5). In this connection, prior to the use and also final testing of the catalyst for indicating its activity in many subsequent runs, the catalyst was recycled from the reaction mixture through a sintered glass funnel (vacuum-filtering). The catalyst was washed successively with THF or acetone $(10 \mathrm{~mL})$ and dried in a vacuum oven at $80^{\circ} \mathrm{C}$ for $30 \mathrm{~min}$.

The catalyst was tested for five consecutive runs and through each run, no fresh catalyst was added. Furthermore, the ICP analysis has confirmed the reusability of
Table 5. The reusability of $\mathrm{SiO}_{2}-\mathrm{Cu}-\mathrm{BDACDABSBC}$ in successive runs for the synthesis of $\mathbf{2} \mathbf{a}^{\mathrm{a}}$

\begin{tabular}{ccc} 
Run no. ${ }^{b}$ & Time (h) & Yield ${ }^{c}(\%)$ \\
\hline 1 & 4 & 94 \\
2 & 4 & 92 \\
3 & 4.5 & 91 \\
4 & 4.5 & 91 \\
5 & 5 & 88 \\
\hline
\end{tabular}

a Reaction conditions: nitrile (0.01 mol), $\mathrm{NaN}_{3}(0.015 \mathrm{~mol})$, recovered $\mathrm{SiO}_{2}-\mathrm{Cu}$-BDACDABSBC, $\mathrm{H}_{2} \mathrm{O} / \mathrm{i}$-PrOH $(50 \mathrm{~mL}) .{ }^{\mathrm{b}}$ The entry number corresponds to the trial number. ${ }^{\mathrm{C}}$ Isolated yield.

the $\mathrm{SiO}_{2}-\mathrm{Cu}-\mathrm{BDACDABSBC}$ without significant desorption of $\mathrm{Cu}$ species from the silica matrix. As it is well indicated, the amount of leached $\mathrm{Cu}$ from $\mathrm{SiO}_{2}$ - $\mathrm{Cu}-\mathrm{BDAC}$ $\mathrm{DABSBC}$ is extremely negligible $(0.06 \%$ after five consecutive runs).

As the results in Table 5 indicate, the catalyst can be reused for many consecutive runs without considerable decrease in its catalytic reactivity.

\section{2. Antifungal Studies}

The antifungal activities of 5 -substituted- $1 \mathrm{H}$-tetrazole derivatives against yeasts and filamentous fungi were evaluated in vitro. The minimal inhibitory concentrations (MICs) of the tested compounds were determined by the micro broth dilution method in 96-well microplates according to the CLSI-M27-A3 and M27-S4 methods for yeasts ${ }^{81-82}$ and CLSI-M38-A2 for filamentous fungi. ${ }^{83} \mathrm{An}$ tifungal agents as quality controls including amphotericin B (AMB) (Bristol-Myers-Squib, Woerden, The Netherlands), itraconazole (ITZ) (Janssen Research Foundation, Beerse, Belgium), voriconazole (VRZ) (Sigma), posaconazole (PSZ) (Sigma) and fluconazole (FLZ) (Pfizer, Groton, CT, USA) were obtained as reagent-grade powders from the respective manufacturers for the preparation of CLSI microdilution trays. The standard isolates as quality controls were obtained from collections of ATCC (American Type Culture Collection); its yeasts consist of: Candida species [C. albicans (ATCC 10231), C. glabrata (ATCC 2001), C. krusei (ATCC 6258), C. parapsilosis (ATCC 22019)] and filamentous fungi consist of Aspergillus species [A. fumigatus (ATCC MYA-2636), A. flavus (ATCC 204304)]. Some tetrazol compounds were dissolved in DMSO and serially diluted in the standard RPMI-1640 medium (Sigma Chemical Co.) and buffered to $\mathrm{pH} 7.0$ with $0.165 \mathrm{M}$-morpholinepropanesulfonic acid (MOPS) buffer (Sigma) and L-glutamine without bicarbonate (maximum concentration was considered $512 \mu \mathrm{g} / \mathrm{mL}$ for 
Table 4. The synthesized new $1 \mathrm{H}$-tetrazoles using $\mathrm{SiO}_{2}-\mathrm{Cu}-\mathrm{BDACDABSBC} \mathrm{C}^{\mathrm{a}}$

\begin{tabular}{|c|c|c|c|c|}
\hline Entry & Nitrile & Product $^{b}$ & Time (h) & Yield $^{c}(\%)$ \\
\hline 1 & & & 4 & 94 \\
\hline 2 & & & 6 & 90 \\
\hline 3 & & & 6 & 75 \\
\hline 4 & & & 3 & 80 \\
\hline 5 & & & 4 & 95 \\
\hline 6 & & & 3 & 82 \\
\hline 7 & & & 6 & 80 \\
\hline 8 & & & 4 & 91 \\
\hline 9 & & & 3 & 79 \\
\hline 10 & & & 8 & 79 \\
\hline 11 & & & 7 & 82 \\
\hline 12 & & & 6 & 85 \\
\hline 13 & & & 5 & 85 \\
\hline 14 & & & 6 & 93 \\
\hline 15 & & & 5 & 90 \\
\hline
\end{tabular}

${ }^{a}$ Reaction conditions: nitrile $(0.01 \mathrm{~mol}), \mathrm{NaN}_{3}(0.015 \mathrm{~mol}), \mathrm{SiO}_{2}$-Cu-BDACDABSBC $(0.05 \mathrm{~mol} \%)$, water $/ i-\mathrm{PrOH}$ $(50 \mathrm{~mL}) .{ }^{\mathrm{b}}$ All products were characterized by ${ }^{1} \mathrm{H}$ and ${ }^{13} \mathrm{C}$ NMR, IR, $\mathrm{CHN}$, and $\mathrm{MS}$ analysis. ${ }^{\mathrm{c}}$ Isolated yield. 
all compounds). Briefly, the inoculum suspension was added to each well and incubated at $35^{\circ} \mathrm{C}$. MIC was defined as the minimum inhibitory concentration of the tested compound which resulted in total inhibition of the fungal growth. All susceptibility testing was performed in duplicate.

Table 6 summarizes the MIC values of 6 standard isolates of yeasts and filamentous fungi of five antifungal drugs and 15 tetrazol compounds. The in vitro susceptibility results obtained for FLZ, ITZ, VRZ, PSZ and AMB against the standard isolates were within the ranges that are considered normal for these strains. ${ }^{84}$

The results of MIC of 15 tetrazol derivatives have shown that the lowest MIC with $64 \mu \mathrm{g} / \mathrm{mL}$ was obtained for five compounds (2g, 2k, 21, 2m and $\mathbf{2 n}$ ) against Aspergillus fumigatus (ATCC MYA-2636), and the highest MIC with 512 and $>512 \mu \mathrm{g} / \mathrm{mL}$ was measured for all compounds against Candida glabrata (ATCC 2001). In addition, the lowest MIC values against all standard isolates were measured for three tetrazols $(\mathbf{2 g}, \mathbf{2 l}$, and $\mathbf{2 n}$ ), and the highest MIC values against all standard isolates for $2 \mathrm{e}$. So, Table 6 shows $\mathrm{MIC}_{50}$ and $\mathrm{MIC}_{90}$ values expressed in $\mu \mathrm{g} / \mathrm{mL}$ for all tetrazole derivatives. The lowest $\mathrm{MIC}_{50}(128 \mu \mathrm{g} / \mathrm{mL})$ was obtained for $\mathbf{2 g}, \mathbf{2 h}, \mathbf{2 i}, \mathbf{2 k}, \mathbf{2}, \mathbf{2} \mathbf{m}$ and $\mathbf{2 n}$ and the highest $\mathrm{MIC}_{50}(512 \mu \mathrm{g} / \mathrm{mL})$ for $2 \mathrm{e}$, thus the lowest $\mathrm{MIC}_{90}(128 \mu \mathrm{g} /$ $\mathrm{mL}$ ) was measured for $\mathbf{2 g}, \mathbf{2 l}$ and $\mathbf{2 n}$ and the highest $\mathrm{MIC}_{90}$ $(>512 \mu \mathrm{g} / \mathrm{mL})$ for $2 \mathbf{e}$ and $\mathrm{MIC}_{90}(512 \mu \mathrm{g} / \mathrm{mL})$ for $\mathbf{2 j}$ and $\mathbf{2 o}$ (Table 6).

\section{3. Molecular Docking Study}

Molecular docking study is a procedure which predicts the interactions of the novel synthetic compound as a drug candidate with the target enzyme and/or receptor binding sites to form a stable complex. ${ }^{85}$ Since $2 \mathrm{n}$ was specified as the most potent antifungal agent, thus the binding mode of $\mathbf{2 n}$ in the active site of cytochrome P450-dependent $14 \alpha$-lanosterol demethylase was investigated carrying out a molecular docking study.

In the case of docking method, every ligand was optimized with different minimization structures, they were thereafter converted to PDBQT using MGL tools 1.5.6. ${ }^{86}$ Co-crystal ligand molecules were excluded from the structures and the PDBs were corrected in terms of missing atom types by modeler $9.12 .{ }^{87} \mathrm{~A}$ house application (MODELFACE) was used for the generation of python script and running modeller software. Consequently, the enzymes were transformed to PDBQT and Gasteiger partial charges were added using MGLTOOLS1.5.6. The docking simulations were achieved by means of an in-house batch script (DOCK-FACE) for automatic running of Auto Dock 4.2, ${ }^{88}$ in a parallel mode, using all system resources.

In all experiments genetic algorithm search technique was applied to find the best pose of each ligand in the active site of the target enzyme. Random orientations of the conformations were obtained after translating the center of the ligand to a specified position within the re-

Table 6. In vitro susceptibility testing of 6 standard isolates of yeasts and filamentous fungi to four antifungal agents and 15 novel compounds, and $\mathrm{MIC}_{50}$ and $\mathrm{MIC}_{90}$ values expressed in $\mu \mathrm{g} / \mathrm{ml}$ for 5 -substituted- $1 H$-tetrazole derivatives ${ }^{\mathrm{a}}$

\begin{tabular}{|c|c|c|c|c|c|c|c|c|}
\hline \multirow[b]{2}{*}{ Compound } & \multirow[b]{2}{*}{$\begin{array}{l}\text { Candida } \\
\text { albicans }\end{array}$} & \multirow[b]{2}{*}{$\begin{array}{l}\text { Candida } \\
\text { glabrata }\end{array}$} & \multirow[b]{2}{*}{$\begin{array}{c}\text { Candida } \\
\text { krusei }\end{array}$} & \multicolumn{2}{|c|}{$\operatorname{MIC}^{b}(\mu \mathrm{g} / \mathrm{mL})$} & \multirow[b]{2}{*}{$\begin{array}{l}\text { Aspergillus } \\
\text { flavus }\end{array}$} & \multirow[b]{2}{*}{$\mathrm{MIC}_{50}$} & \multirow[b]{2}{*}{$\mathrm{MIC}_{90}$} \\
\hline & & & & $\begin{array}{c}\text { Candida } \\
\text { parapsilosis }\end{array}$ & $\begin{array}{l}\text { Aspergillus } \\
\text { fumigatus }\end{array}$ & & & \\
\hline $2 a$ & 256 & 512 & 256 & 512 & 128 & 256 & 256 & 256 \\
\hline $2 b$ & 256 & 512 & 256 & 256 & 128 & 256 & 256 & 256 \\
\hline $2 c$ & 256 & 512 & 256 & 256 & 128 & $>512$ & 256 & 256 \\
\hline $2 \mathrm{~d}$ & 256 & 512 & 256 & 256 & 128 & $>512$ & 256 & 256 \\
\hline $2 \mathrm{e}$ & 512 & $>512$ & 512 & 512 & $>512$ & $>512$ & 512 & $>512$ \\
\hline $2 \mathrm{f}$ & 256 & 512 & 256 & 256 & 128 & 256 & 256 & 256 \\
\hline $2 \mathrm{~g}$ & 128 & 256 & 128 & 128 & 64 & 128 & 128 & 128 \\
\hline $2 \mathrm{~h}$ & 128 & 256 & 128 & 128 & 128 & 256 & 128 & 256 \\
\hline $2 \mathrm{i}$ & 256 & 512 & 128 & 256 & 128 & 256 & 128 & 256 \\
\hline $2 \mathrm{j}$ & 256 & 512 & 256 & 256 & 256 & $>512$ & 256 & 512 \\
\hline $2 \mathrm{k}$ & 128 & 256 & 128 & 128 & 64 & 256 & 128 & 256 \\
\hline 21 & 128 & 256 & 128 & 128 & 64 & 128 & 128 & 128 \\
\hline $2 \mathrm{~m}$ & 128 & 512 & 256 & 128 & 64 & 256 & 128 & 256 \\
\hline $2 n$ & 128 & 256 & 128 & 128 & 64 & 128 & 128 & 128 \\
\hline 20 & 256 & 512 & 256 & 256 & $>512$ & 256 & 256 & 512 \\
\hline Fluconazole ${ }^{c}$ & 0.25 & 32 & 64 & 0.125 & 2 & 2 & - & - \\
\hline Itraconazole ${ }^{c}$ & 0.25 & 0.25 & 0.25 & 0.063 & 1 & 0.5 & - & - \\
\hline Voriconazole $^{c}$ & 0.125 & 0.25 & 0.125 & 0.031 & 0.5 & 0.5 & - & - \\
\hline Posaconazole $^{c}$ & 0.125 & 0.25 & 0.125 & 0.031 & 0.5 & 0.25 & - & - \\
\hline Amphotricin $B^{c}$ & 0.5 & 0.5 & 0.5 & 0.063 & 1 & 1 & - & - \\
\hline
\end{tabular}

\footnotetext{
${ }^{a}$ Examined fungi: Candida albicans (ATCC 10231), C. krusei (ATCC 6258), C. glabrata (ATCC 2001), C. parapsilosis (ATCC 22019), Aspergillus
} fumigatus (ATCC MYA-2636) and A. flavus (ATCC 204304) ${ }^{\mathrm{b}}$ Minimal inhibitory concentration ${ }^{\mathrm{c}}$ Reference drugs for fungal species 
ceptor active site, and making a series of rotamers. This process was recursively repeated until the desired number of low energy orientations was obtained. Cluster analysis was performed on the docked results using a root mean square deviation (RMSD) tolerance of $1.8 \AA$. For the internal validation phase, ligand inside the pdb file of aromatase (1ea1) was extracted using a viewer and treated the same as other ligands in this study.

It is well known that the azoles as antifungal agents are able to inhibit CYP51 through the binding to $\mathrm{N}$-atoms in azoles with the iron core inside the haem. To accredit the docking protocol, fluconazole was redocked in the active site of Mycobacterium P450DM (Figure 2). As can be seen, the substrate-binding pocket of Mycobacterium

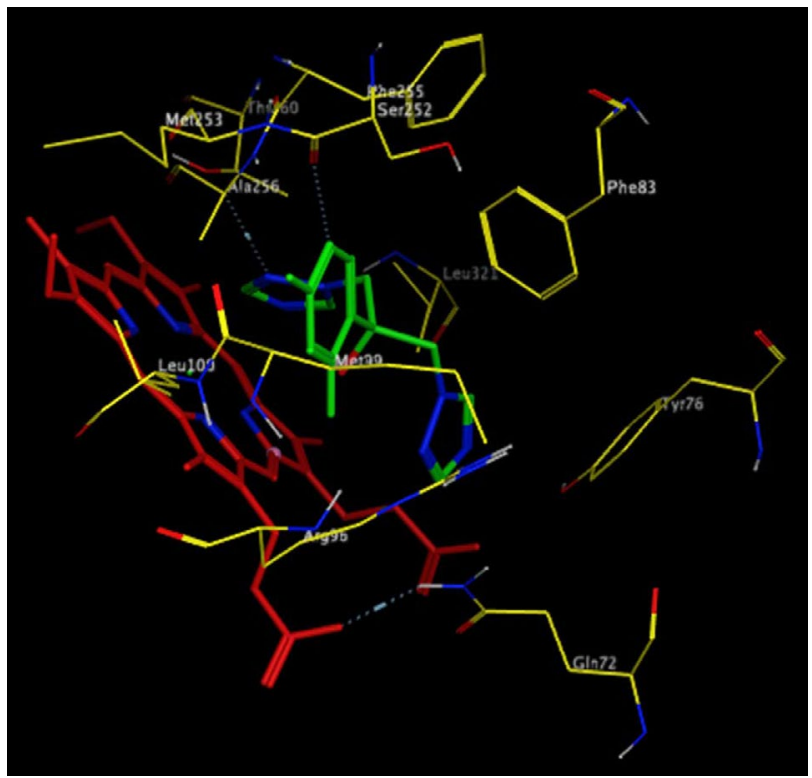

Figure 2. Docking conformation of fluconazole at the active site of Mycobacterium P450DM

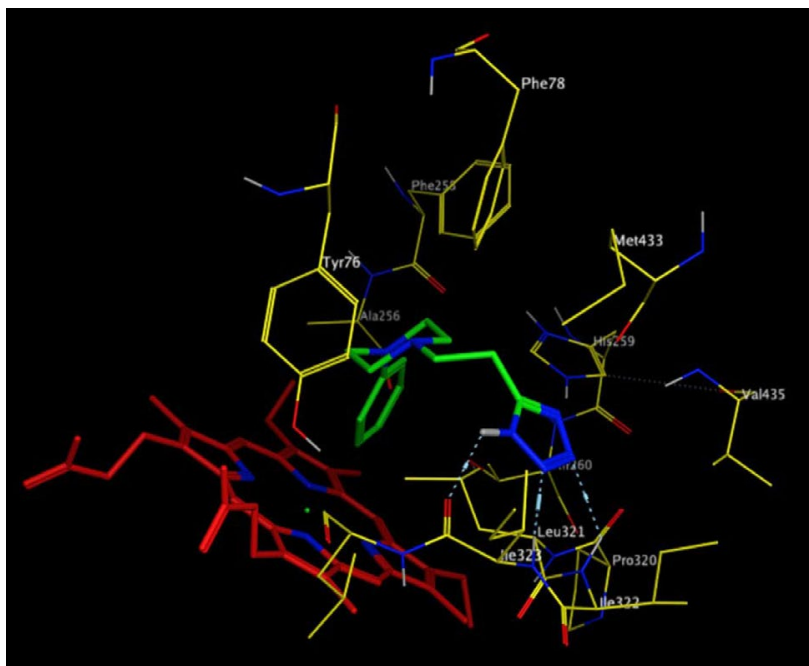

Figure 3. Docking conformation of $\mathbf{2 n}$ at the active site of Mycobacterium P450DM
$\mathrm{P} 450 \mathrm{DM}$ is above the porphyrin ring with the ceiling lipophilic amino acid residues (Phe78, Met79, Phe83, and Phe255). Moreover, access to the pyrrole rings is restricted by Thr260, Ala256, and Leu321, respectively. ${ }^{89}$ Just as it was found with fluconazole, compound $\mathbf{2 n}$ is accommodated at the same binding site and showed a strong interaction with Mycobacterium P450DM enzyme (Figure 3).

Interestingly, similarly to fluconazole, $\mathbf{2 n}$ is incorporated in the same binding site and showed a strong interaction with the enzyme active site. The calculated bonding energy values for fluconazole and $\mathbf{2 n}$ are -8.10 and -9.22 $\mathrm{kcal} / \mathrm{mol}$, respectively, indicating there is an energy gap of about $1.12 \mathrm{kcal} / \mathrm{mol}$. To this energy gap the stronger binding of $2 \mathrm{n}$ at the active site of the enzyme can be attributed. As can be seen in Figure 2, which clearly shows the hydrogen bondings of $\mathrm{N}^{1}-\mathrm{H}$ tetrazol with oxygen in Leu324, this hydrogen bonding plays an important role in the higher affinity of $2 \mathbf{n}$ to the active site of the Mycobacterium P450DM enzyme. The aliphatic side chain tethered to the tetrazol ring is bound in the hydrophobic pocket above the haem group with residues including Ile323 and Ile 322.

\section{Conclusions}

In conclusion, we have explained a recyclable heterogeneous catalysts that was used in synthesis of some 5-substituted $1 \mathrm{H}$-tetrazoles bearing bioactive $\mathrm{N}$-heterocyclic cores. The main advantages of this methodology are its simplicity of the reaction procedure, mild reaction condition, and good to excellent yields. Furthermore, copper(II) catalyst can be recovered and recycled by simple filtration of the reaction mixture and reused for at least five consecutive trials without significant loss of its activity. The antifungal tests have shown antifungal activity against all groups of fungal for some compounds. The docking analysis has demonstrated the appropriate fitting of $\mathbf{2 n}$ in active site of Mycobacterium P450DM enzyme.

\section{Acknowledgement}

We are grateful to Fasa University of Medical Sciences for their financial support.

\section{References}

1. R. N. Butler, in: A. R. Katritzky, C. W. Rees, E. F. V. Scriven (Ed.): Comprehensive Heterocyclic Chemistry, Pergamon, Oxford, 1996, 4, pp. 1-126.

2. H. Bräuner-Osborne, J. Egebjerg, E. Ø. Nielsen, U. Madsen, P. Krogsgaard-Larsen, J. Med. Chem. 2000, 43, 2609-2645.

DOI:10.1021/jm000007r

3. J. M. Essery, J. Med. Chem. 1996, 12, 703-705.

DOI:10.1021/jm00304a039 
4. A. Andrus, B. Partridge, J. V. Heck. B. G. Christensen, Tetrahedron Lett. 1984, 25, 911-1917.

DOI:10.1016/S0040-4039(01)80060-5

5. V. Dhayanithhi, S. S. Syed, K. Kumaran, K. R. J. Sankar, R. V. Ragavan, P. S. K. Goud, N. S. Kumari, H. N. Pati, J. Serb. Chem. Soc. 2011, 76, 165-175. DOI:10.2298/JSC090421001D

6. R. S. Upadhayaya, S. Jain, N. Sinha, N. Kishore, R. Chandra, S. K. E. Arora, Eur. J. Med. Chem. 2004, 39, 579-592.

DOI:10.1016/j.ejmech.2004.03.004

7. A. Rajasekaran, N. Sankar, A. Murugesh, A. R. Kalasalingam, Arch. Pharm. Res. 2006, 29, 535-540.

DOI:10.1007/BF02969261

8. P. B. Mohite, R. B. Pandhare, S. G. Khanage, V. H. Bhaskar, Adv. Pharm. Bull. 2012, 2, 31-36.

9. J. Adamec, K. Waisser, J. Kunes, J. Kaustova, J. Arch. Pharm. 2005, 338, 385-389. DOI:10.1002/ardp.200400967

10. A. O. De Souza, M. T. Pedrosa, J. B. Alderete, A. F. Cruz, M. A. Prado, R. B. Alves, C. L. Silva, Pharmazie 2005, 60, 396-397.

11. H. Akimoto, K. Ootsu, F. Itoh, Eur. Patent EP 530537; Chem. Abstr. 1993, 119, 226417.

12. G. P. Ellis, D. Shaw, J. Med. Chem. 1972, 15, 865-867. DOI:10.1021/jm00278a027

13. E. Vieira, S. Huwyler, S. Jolidon, F. Knoflach, V. Mutel, J. Wichmann, J. Bioorg. Med. Chem. Lett. 2005, 15, 4628-4631. DOI:10.1016/j.bmcl.2005.05.135

14. A. Gagnon, S. Landry, R. Coulombe, A. Jakalian, I. Guse. B. Thavonekham, P. R. Bonneau, C. Yoakim, B. Simoneau, Bioorg. Med. Chem. Lett. 2009, 19, 1199-1205.

DOI:10.1016/j.bmcl.2008.12.074

15. P. N. Gaponik, S. V. Voitekhovich, O. A. Ivashkevich, Russ. J. Chem. Rev. 2006, 75, 507-539.

DOI:10.1070/RC2006v075n06ABEH003601

16. R. J. Herr, Bioorg. Med. Chem. 2002, 10, 3379-3393. DOI:10.1016/S0968-0896(02)00239-0

17. G. I. Koldobskii, V. A. Ostrovskii, Usp. Khim. 1994, 63, 847865. DOI:10.1070/RC1994v063n10ABEH000119

18. J. M. McManus, R. M. Herbst, J. Org. Chem. 1959, 24, 14641467. DOI:10.1021/jo01092a021

19. V. Rama, K. Kanagaraj, K. Pitchumani, J. Org. Chem. 2011, 76, 9090-9095. DOI:10.1021/jo201261w

20. V. A. Ostrovskii, R. E. Trifonov, E. A. Popova, Russ. Chem. Bull. 2012, 61, 768-780. DOI:10.1007/s11172-012-0108-4

21. B. Dahlof, R. B. Deveruex, S. E. Kjeldsen, Lancet 2002, 359, 995-1003. DOI:10.1016/S0140-6736(02)08089-3

22. T. Mosmann, J. Immunol. Methods 1983, 65, 55-63. DOI:10.1016/0022-1759(83)90303-4

23. Z. P. Demko, K. B. Sharpless, Org. Lett. 2001, 3, 4091-4094. DOI:10.1021/ol010220x

24. A. R. Katritzky, C. Cai, N. K. Meher, Synthesis 2007, 8, 12041208. DOI:10.1055/s-2007-966001

25. T. Jin, F. Kitahara, S. Kamjio, Y. Yamamoto, Tetrahedron Lett. 2008, 49, 2824-2827. DOI:10.1016/j.tetlet.2008.02.115

26. D. P. Matthews, J. E. Green, A. J. Shuker, J. Comb. Chem. 2000, 2, 19-23. DOI:10.1021/cc990035z

27. A. Kumar, R. Narayanan, H. Shechter, J. Org. Chem. 1996, 61, 4462-4465. DOI:10.1021/jo952269k
28. Y. S. Gyoung, J. G. Shim, Y. Yamamoto, Tetrahedron Lett. 2000, 41, 4193-4196. DOI:10.1016/S0040-4039(00)00563-3

29. W. K. Su, Z. Hong, W. G. Shan, X. X. Zhang, Eur. J. Org. Chem. 2006, 12, 2723-2726. DOI:10.1002/ejoc.200600007

30. S. Hajra, D. Sinha, M. Bhowmick, J. Org. Chem. 2007, 72, 1852-1855. DOI:10.1021/jo062432j

31. B. Akhlaghinia, S. Rezazadeh, J. Braz. Chem. Soc. 2012, 23, 2197-2203. DOI:10.1590/S0103-50532013005000005

32. Y. Yao, Y. Zhou, B. Lin, C. Yao, Tetrahedron Lett. 2013, 54, 6779-6781. DOI:10.1016/j.tetlet.2013.10.019

33. M. Nasrollahzadeh, Y. Bayat, D. Habibi, S. Moshaee, Tetrahedron Lett. 2009, 50, 4435-4438.

DOI:10.1016/j.tetlet.2009.05.048

34. L. Lang, B. Li, W. Liu, L. Jiang, Z. Xu, G. Yin, Chem. Commun. 2010, 46, 448-450. DOI:10.1039/B912284B

35. M. Lakshim Kantam, V. Balasubramanyam, K. B. Shiva Kumar, Synth. Commun. 2006, 36, 1809-1814.

DOI:10.1080/00397910600619630

36. G. Aridoss, K. K. Laali, Eur. J. Org. Chem. 2011, 52, 63436355. DOI:10.1002/ejoc.201100957

37. M. Lakshmi Kantam, K. B. Shiva Kumar, K. Phani Raja, J. Mol. Catal. A 2006, 247, 186-188.

DOI:10.1016/j.molcata.2005.11.046

38. G. Venkateshwarlu, K. C. Rajanna, P. K. Saiprakash, Synth. Commun. 2009, 39, 426-432.

DOI:10.1080/00397910802378381

39. J. He, B. Li, F. Chen, Z. Xu, G. Yin, J. Mol. Catal. A 2009, 304, 135-138. DOI:10.1016/j.molcata.2009.01.037

40. G. Venkateshwarlu, A. Premalatha, K. C. Rajanna, P. K. Saiprakash, Synth. Commun. 2009, 39, 4479-4485.

DOI:10.1080/00397910902917682

41. G. Qi, Y. Dai, Chin. Chem. Lett. 2010, 21, 1029-1032. DOI:10.1016/j.cclet.2010.05.003

42. M. Nasrollahzadeh, D. Habibi, Z. Shahkarami, Y. Bayat, Tetrahedron 2009, 65, 10715-10719.

DOI:10.1016/j.tet.2009.10.029

43. M. N. Soltani Rad, S. Behrouz, M. Doroodmand, A. Movahedian, Tetrahedron 2012, 68, 7812-7821.

DOI:10.1016/j.tet.2012.07.032

44. A. Corma, H. Garcia, Adv. Synth. Catal. 2006, 348, 13911412. DOI:10.1002/adsc.200606192

45. S. Minakata, M. Komatsu, Chem. Rev. 2009, 109, 711-724. DOI: $10.1021 / \mathrm{cr} 8003955$

46. C. A. McNamara, M. J. Dixon, M. Bradley, Chem. Rev. 2002, 102, 3275-3300. DOI:10.1021/cr0103571

47. H. Hirai, N. Ohtsuka, T. Shimazawa, React. Funct. Polym. 1998, 37, 199-212. DOI:10.1016/S1381-5148(97)00170-3

48. T. Toupance, M. Kermarec, C. Louis, J. Phys. Chem. B 2000, 104, 965-972. DOI:10.1021/jp993399q

49. N. L. Dias Filho, Y. Gushikem, D. W. Franco, M. S. Schultz, L. C. G. Vasconcellos, Colloids Surf., A 1998, 141, 181-187. DOI:10.1016/S0927-7757(98)00333-1

50. F. Cozzi, Adv. Synth. Catal. 2006, 348, 1367-1390. DOI:10.1002/adsc.200606096

51. K. Binnemans, Chem. Rev. 2009, 109, 4283-4374. DOI: $10.1021 / \mathrm{cr} 8003983$ 
52. C. Baleizão, H. Garcia, Chem. Rev. 2006, 106, 3987-4043. DOI:10.1021/cr050973n

53. A. F. Trindade, P. M. P. Gois, C. A. M. Afonso, Chem. Rev. 2009, 109, 418-514. DOI:10.1021/cr800200t

54. J. M. Fraile, J. I. García, J. A. Mayoral, Chem. Rev. 2009, 109, 360-417. DOI:10.1021/cr800363y

55. A. P. Wight, M. E. Davis, Chem. Rev. 2002, 102, 3589-3614. DOI: $10.1021 / \mathrm{cr} 020068 \mathrm{~s}$

56. L. Yin, J. Liebscher, Chem. Rev. 2007, 107, 133-173. DOI:10.1021/cr0505674

57. J. Miłobędzka, S. von Kostanecki, V. Lampe, Ber. Dtsch. Chem. Ges. 1910, 43, 2163-2170. DOI:10.1002/cber.191004302168

58. R. Waranyoupalin, S. Wongnawa, M. Wongnawa, C. Pakawatchai, P. Panichayupakaranant, P. Sherdshoopongse, Cent. Eur. J. Chem. 2009, 7, 388-394.

DOI:10.2478/s11532-009-0037-8

59. A. Goel, A. B. Kunnumakkara, B. B. Aggarwal, Biochem. Pharmacol. 2009, 75,787-809.

DOI:10.1016/j.bcp.2007.08.016

60. T. Ueno, M. Ohashi, M. Kono, K. Kondo, A. Suzuki, T. Yamane, Y. Watanabe, Inorg. Chem. 2004, 43, 2852-2858.

DOI:10.1021/ic0498539

61. S. Pal, A. K. Barik, S. Gupta, A. Hazra, S. K. Kar, S. M. Peng, G. H. Lee, R. J. Butcher, M. S. E. I. Fallah, J. Ribas, Inorg. Chem. 2005, 44, 3880-3889. DOI:10.1021/ic0501420

62. M. Babazadeh-Qazijahani, H. Badali, H. Irannejad, M. H. Afsarian, S. Emami, Eur. J. Med. Chem. 2014, 76, 264-273. DOI:10.1016/j.ejmech.2014.02.019

63. S. M. H. Afsarian, H. Badali, T. Shokohi, S. Najafipour, Iran. J. Public Health 2015, 44, 1262.

64. M. H. Afsarian, H. Badali, T. Boekhout, T. Shokohi, F. Katiraee, J. Med. Microbiol. 2015, 64, 248-253.

DOI:10.1099/jmm.0.000015

65. S. M. Hashemi, H. Badali, M. A. Faramarzi, N. Samadi, M. H. Afsarian, Mol. Divers. 2015, 19, 15-27.

DOI:10.1007/s11030-014-9548-0

66. M. A. Pfaller, Am. J. Med. 2012, 125, 3-13.

DOI:10.1016/j.amjmed.2011.11.001

67. X. Chai, J. Zhang, Y. Cao, Y. Zou, Q. Wu, D. Zhang, Eur. J. Med. Chem. 2012, 46, 3142-3148.

DOI:10.1016/j.ejmech.2011.02.042

68. Y. Y. Zhang, J. L. Mi, C. H. Zhou, X. D. Zhou, Eur. J. Med. Chem. 2011, 46, 4391-402. DOI:10.1016/j.ejmech.2011.07.010

69. T. Shokohi, H. Badali, N. Amirrajab, M. R. Ataollahi, S. A. Kouhpayeh, M. H. Afsarian, Iran. Curr. Med. Mycol. 2016, 2, 34-39. DOI:10.18869/acadpub.cmm.2.2.8

70. M. A. Asif, J. Bioorg. Chem. 2017, 2, 146-152.

71. S. Esmaielzadeh, E. Zarenezhad, Acta Chim. Slov. 2018, 65, 416-428. DOI:10.17344/acsi.2018.4159

72. E. Zarenezhad, M. N. Soltani Rad, S. Behrouz, S. Esmaielzadeh, M. Farjam, J. Iran. Chem. Soc. 2017, 14, 509-519.

DOI:10.1007/s13738-016-0999-3
73. S. Behrouz, M. N. Soltani Rad, S. Rostami, M. Behrouz, E. Zarehnezhad, A. Zarehnezhad, Mol. Divers. 2014, 18, 797-808. DOI:10.1007/s11030-014-9539-1

74. M. N. Soltani Rad, S. Behrouz, M. Behrouz, A.Sami, M. Mardkhoshnood, A. Zarenezhad, E. Zarenezhad, Mol. Divers. 2016, 20, 705-718. DOI:10.1007/s11030-016-9678-7

75. M. N. Soltani Rad, S. Behrouz, E. Zarenezhad, M. H. Moslemin, A. Zarenezhad, M. Mardkhoshnood, M. Behrouz, S. Rostami, Med. Chem. Res. 2014, 23, 3810-3822.

DOI:10.1007/s00044-014-0967-3

76. M. N. Soltani Rad, S. Behrouz, E. Zarenezhad, N. Kaviani, J. Iran. Chem. Soc. 2015, 12, 1603-1612. DOI:10.1007/s13738-015-0633-9

77. E. Zarenezhad, M. N. Soltani Rad, M. H. Mosslemin, M. Tabatabaee, S. Behrouz, J. Chem. Res. 2014, 38, 607-610. DOI:10.3184/174751914X14115772243815

78. M. H. Mosslemin, E. Zarenezhad, N. Shams, M. N. Soltani Rad, J. Chem. Res. 2014, 38, 169-171. DOI:10.3184/174751914X13917105358323

79. M. N. Soltani Rad, S. Behrouz, V. Sadeghi Dehchenari, S. J. Hoseini, J. Heterocycl. Chem. 2017, 54, 355-365.

DOI:10.1002/jhet.2777

80. J. Rajesh, A. Gubendran, G. Rajagopal, P. Athappan, J. Mol. Struct. 2012, 1010, 169-178.

DOI:10.1016/j.molstruc.2011.12.002

81. P. A. Wayne: Reference method for broth dilution antifungal susceptibility testing of yeasts, approved standard. CLSI document M27-A2, 2002.

82. P. Wayne: Clinical and laboratory standards institute. Implementation Guide of POCT for health care providers, 2006, $1-37$.

83. Clinical Institute LS. Reference Method for Broth Dilution Antifungal Susceptibility Testing of Filamentous Fungi: Approved Standard. CLSI document M38-A2, 2008.

84. A. L. Barry, M. A. Pfaller, S. D. Brown, A. Espinel-Ingroff, M. A. Ghannoum, C. Knapp, R. P. Rennie, J. H. Rex, M. G. Rinaldi, J. Clin. Microbiol. 2000, 38, 3457-3459.

85. T. Lengauer, M. Rarey, Curr. Opin. Struct. Biol. 1996, 6, 402406. DOI:10.1016/S0959-440X(96)80061-3

86. G. M. Morris, R. Huey, A. J. Olson: Using autodock for ligand-receptor docking. Current Protocols in Bioinformatics. 2008, 24, 8-14. DOI:10.1002/0471250953.bi0814s24

87. N. Eswar, B. Webb, M. A. Marti-Renom, M. S. Madhusudhan, D. Eramian, M. Y. Shen, U. Pieper, A. Sali, Comparative protein structure modeling using Modeller. Current Protocols in Bioinformatics. 2006, 15, 5-6.

DOI:10.1002/0471250953.bi0506s15

88. A. Sakhteman: PreAuposSOM, https:// www.biomedicale. univ-paris5.fr/aupossom/.

89. L. M. Podust, T. L. Poulos, M. R. Waterman, Proc. Natl. Acad. Sci. U. S. A. 2001, 98, 3068-3073.

DOI: $10.1073 /$ pnas.061562898

Except when otherwise noted, articles in this journal are published under the terms and conditions of the Creative Commons Attribution 4.0 International License 


\section{Povzetek}

$\mathrm{V}$ članku opisujemo enostavno sintezno pot, ki temelji na [3+2] cikloadiciji alkil nitrilov (RCN) z natrijevim azidom $\left(\mathrm{NaN}_{3}\right)$ v prisotnosti bakrovega kompleksa $\mathrm{z}$ bis(diacetilkurkumin) 1,2-diaminobenzensko Schiffovo bazo, ki je imobiliziran na silikagelu: $\mathrm{SiO}_{2}$ - $[\mathrm{Cu}-\mathrm{BDACDABSBC}]$, ter igra vlogo heterogenega katalizatorja, in askorbinske kisline $\mathrm{v}$ zmesi topil vode in $i-\mathrm{PrOH}(50: 50, \mathrm{~V} / \mathrm{V})$ pod pogoji refluksa. Katalizator na nosilcu smo pripravili z imobilizacijo bakrovega kompleksa bis(diacetilkurkumin) 1,2-diaminobenzenske Schiffove baze [Cu-BDACDABSBC] na silikagel. Ta kompleks se je izkazal kot visokoselektiven katalizator z veliko aktivnostjo in z dobro možnostjo recikliranja. Glavne odlike opisane sinteze so visoki izkoristki, široka paleta možnih izhodnih spojin ter enostavna in učinkovita izolacija, kar je omogočilo pripravo 5-substituiranih $1 H$-tetrazolov, ki so vključevali $N$-heterociklične bioaktivne sisteme, z odličnimi izkoristki. Za pripravljene spojine smo in vitro določili protiglivično učinkovanje na nekatere vrste patogenih gliv iz rodu Candida ( $C$. albicans, C. glabrata, C. krusei in C. parapsilosis) ter na nekatere filamentne glive iz rodu Aspergillus (A. fumigatus in A. flavus). Študije molekulskega sidranja smo izvedli za najbolj učinkovito izmed pripravljenih spojin, kar nam je omogočilo, da smo razvoljali izjemno interakcijo med to spojino in aktivnim mestom Mycobacterium P450DM. 